\title{
Extension of the Intranuclear Cascade Model for Photonuclear Reactions at Energies up to $10 \mathrm{GeV}$
}

\author{
A.S. Iljinov, I.A. Pshenichnov \\ Institute for Nuclear Research, Russian Academy of Science, 117312 Moscow, Russia \\ N. Bianchi, E. De Sanctis, V. Muccifora, M. Mirazita, P. Rossi \\ INFN-Laboratori Nazionali di Frascati, P.O. Box 13, I-00044 Frascati (Rome), Italy
}

\begin{abstract}
The intranuclear cascade (INC) model for photonuclear reactions has been extended at energies up to $10 \mathrm{GeV}$. To this end a phenomenological method for exclusive description of the elementary $\gamma N$-interaction has been developed taking into account both the resonance contribution from the two-body channels $\gamma N \rightarrow \pi B^{\star}$ and $\gamma N \rightarrow M^{\star} N$ ( $B^{\star}$ and $M^{\star}$ being baryon and meson resonances) and the non-resonant statistical contribution from multi-body channels $\gamma N \rightarrow i \pi N(2 \leq i \leq 8)$. This method well describes a wide range of $\gamma N$ data up to $E_{\gamma} \sim 10 \mathrm{GeV}$. The INC model takes into account rescattering and absorption in a nucleus of pions, nucleons, and $\eta_{-}^{-}, \rho_{-}^{-}, \omega$-mesons generated in the $\gamma N$-interaction. The evaporation, fission and multifragmentation of highly excited residual nucleus have also been taken into account. As a result of the Monte Carlo simulation an event of $\gamma A$-reaction is generated which contains mesons, nucleons, nuclear fragments, fission fragments and final nuclide. The model well describes the available data on meson production and proton emission obtained with quasi-monochromatic photons.
\end{abstract}

PACS.: 25.20.Lj; 13.60.Le, 13.60.Rj

Submitted to

Nuclear Physics A 


\section{Introduction}

The new continuous-wave high-intensity electron accelerator CEBAF at TJNAF opens new prospects in the experimental study of the interaction of photons with nuclei at energies up to $6 \mathrm{GeV}$. In particular, it allows to investigate in detail processes like the interaction of multi-pion system with a nucleus, the production and propagation of baryon and meson resonances in nuclei, the production of strangeness and hypernuclei, the onset of the shadowing effect, the formation of hot nuclei, and other phenomena. To study these processes one needs models for photonuclear reaction which completely describe multi-body final states. The only exclusive model presently available is the intranuclear cascade (INC) model which has been used to describe a wide range of experimental data [1]-[4]. In Ref.[1] the INC model took into account the channels of $\gamma N$-interaction with production of one and two pions, and then could be applied at photon energy up to $1 \mathrm{GeV}$. In Refs. [2],[3] it was restricted to lower energy, $E_{\gamma} \leq 0.5 \mathrm{GeV}$, as it took only into account the photoproduction process $\gamma N \rightarrow \pi N$.

The aim of the present paper is to extend the range of applicability of the INC model [1] to photonuclear reactions up to the energy $E_{\gamma} \sim 10 \mathrm{GeV}$ and to improve it in the energy range $0.5 \leq E_{\gamma} \leq 1.5 \mathrm{GeV}$. In section 2 we give a brief description of the main assumption of the model. There we present in detail the phenomenological method which describes exclusive production of 2 to 8 pions in the elementary $\gamma N$-interaction. The method takes into account both the resonance contribution (via production and decay of baryon $\Delta, \mathrm{N}^{\star}$ and meson $\eta, \rho, \omega$ resonances), and the non-resonant statistical contribution to the pion production. In Section 3 we present a comparison between the experimental data on $\gamma N$-interaction and the results obtained with the $\gamma N$-event generator which demonstrates its accuracy. In Section 4 we describe the $\gamma A$-event generator based on the INC model in which the fast hadrons produced in a primary $\gamma N$-process interact with the nucleus. In Section 5 we compare the results of $\gamma A$-event generator with the experimental data obtained with quasi-monochromatic photons. Conclusions contain the hints for further development on INC model.

\section{The $\gamma N$-event generator: model and calculation technique}

It is well known that the characteristics of photonuclear interaction is determined by the photon de Broglie wavelength $\lambda$. In the energy range $50 \leq E_{\gamma} \leq 150$ $\mathrm{MeV}$ (below the pion production threshold), $\lambda$ is comparable to the internucleonic distance in a nucleus, and absorption of a photon by a neutron-proton pair is the main mechanism. At higher energies, when $\downarrow$ becomes comparable or even smaller than the nucleon radius, a photon interacts, mainly, with an individual intranuclear nucleon.

The main process of the single-nucleon photoabsorption mechanism is the pion 
production on a nucleon. ${ }^{1}$ The list of channels of elementary $\gamma N$-interaction which we have taken into account in the INC model is given in Table 1.

\begin{tabular}{|c|c|}
\hline$\gamma p$-interaction & $\gamma n$-interaction \\
\hline$\gamma p \rightarrow \pi^{+} n$ & $\gamma n \rightarrow \pi^{-} p$ \\
$\gamma p \rightarrow \pi^{0} p$ & $\gamma n \rightarrow \pi^{0} n$ \\
$\gamma p \rightarrow \pi^{-} \Delta^{++}$ & $\gamma n \rightarrow \pi^{-} \Delta^{+}$ \\
$\gamma p \rightarrow \pi^{0} \Delta^{+}$ & $\gamma n \rightarrow \pi^{0} \Delta^{0}$ \\
$\gamma p \rightarrow \pi^{+} \Delta^{0}$ & $\gamma n \rightarrow \pi^{+} \Delta^{-}$ \\
$\gamma p \rightarrow \eta p$ & $\gamma n \rightarrow \eta n$ \\
$\gamma p \rightarrow \omega p$ & $\gamma n \rightarrow \omega n$ \\
$\gamma p \rightarrow \rho^{0} p$ & $\gamma n \rightarrow \rho^{0} n$ \\
$\gamma p \rightarrow \rho^{+} n$ & $\gamma n \rightarrow \rho^{-} p$ \\
$\gamma p \rightarrow \pi^{+} \pi^{-} p$ & $\gamma n \rightarrow \pi^{+} \pi^{-} n$ \\
$\gamma p \rightarrow \pi^{0} \pi^{+} n$ & $\gamma n \rightarrow \pi^{0} \pi^{-} p$ \\
$\gamma p \rightarrow \pi^{0} \pi^{0} \pi^{0} p$ & $\gamma n \rightarrow \pi^{0} \pi^{0} \pi^{0} n$ \\
$\gamma p \rightarrow \pi^{+} \pi^{-} \pi^{0} p$ & $\gamma n \rightarrow \pi^{+} \pi^{-} \pi^{0} n$ \\
$\gamma p \rightarrow \pi^{+} \pi^{0} \pi^{0} n$ & $\gamma n \rightarrow \pi^{-} \pi^{0} \pi^{0} p$ \\
$\gamma p \rightarrow \pi^{+} \pi^{+} \pi^{-} n$ & $\gamma n \rightarrow \pi^{+} \pi^{-} \pi^{-} p$ \\
$\gamma p \rightarrow i \pi N(4 \leq i \leq 8)$ & $\gamma n \rightarrow i \pi N(4 \leq i \leq 8)$ \\
$(35$ channels $)$ & $(35$ channels \\
\hline
\end{tabular}

Table 1: Channels of elementary $\gamma N$ interaction taken into account in the INC model.

The two-body channel $\gamma N \rightarrow \pi N$ dominates up to $E_{\gamma} \sim 0.5 \mathrm{GeV}$ while at energies $0.5 \leq E_{\gamma} \leq 2 \mathrm{GeV}$. the channels $\gamma N \rightarrow 2 \pi N$ and $\gamma N \rightarrow 3 \pi N$ play a major role. However in the last energy region a part of the two-body subchannels $\gamma N \rightarrow \pi B^{\star}$ and $\gamma N \rightarrow M^{\star} N$ (being $B^{\star}$ and $M^{\star}$ baryon and meson resonances, respectively) is prominent. ${ }^{2}$

Finally, when the photon energy reaches the value of several $\mathrm{GeV}$, the multiple pion production becomes the main process. A large number $(\sim 80)$ of many-body subchannels is the specific feature of this process.

\subsection{Two-body subprocesses}

To describe the two-body channels we have basically used the Monte Carlo event generator of Corvisiero et al.[6], where we modified the integral cross sections taking

\footnotetext{
${ }^{1}$ The summed cross section of channels with strange particle production amounts only to a few percent of the total hadronic $\gamma N$ cross section [5], therefore this subprocess was neglected.

${ }^{2}$ At $E_{\gamma} \leq 2 \mathrm{GeV}$ the cross section of the two-body subchannels $\gamma N \rightarrow M^{\star} B^{\star}$ is less than the cross section of subchannels $\gamma N \rightarrow \pi B^{\star}$ and $\gamma N \rightarrow M^{\star} N[5]$, and we neglected it.
} 
into account the new data available after its publication and also the old data which were not considered in it. The reason of this was to improve the description of the total $\gamma p$ and $\gamma n$ cross sections (which are known with a high precision) in particular in the region $E_{\gamma} \geq 1 \mathrm{GeV}$, where the original Monte Carlo code of Corvisiero et al. was not very accurate (as it will be shown in Fig.8).

Here we briefly mention the main statements of the calculation of each two-body reaction, describe the modifications we performed, and compare the results of the calculation with the experimental data in order to show the accuracy of our version of the event generator.

The channel $\gamma N \rightarrow \pi N$ was calculated in the framework of the phenomenological approach described in Ref. [7] which can be used up to $E_{\gamma}=1.7 \mathrm{GeV}$. Existing data [8] were interpolated at higher $E_{\gamma}$ values. In this approach, the amplitude of pion photoproduction contains Breit-Wigner resonant terms, Born terms, and a weakly-energy dependent "background" contribution, which is used as an adjustable parameter. The masses and widths of the resonances were taken from the $\pi \mathrm{N}$-scattering data, and their amplitudes were free parameters. Excitation of six baryon resonances were taken into account: among them the $\Delta(1232), N^{\star}(1520)$, and $N^{\star}(1680)$ are the most important. The calculation describes well both the integral [5] (Fig.1) and differential [9]-[13] (Fig. 2) cross sections of $\gamma p \rightarrow \pi^{+} n, \gamma n \rightarrow \pi^{-} p$ and $\gamma p \rightarrow \pi^{0} p$ reactions, and this provided a reliable way to extrapolate results on the case of $\gamma n \rightarrow \pi^{0} n$ reaction where data are not available.

In the original version of the $\gamma N$-event generator [6] the channel $\gamma N \rightarrow \pi \Delta$, with different charge states of the $\pi \Delta$-pair, was calculated in the framework of the phenomenological approach described in Ref.[14]. The Feynman diagram which corresponds to the "contact" interaction, the pole diagram, and the diagram with $D_{13}(1520)$ and $F_{15}(1688)$ s-channel resonances were taken into account in the calculation of the integral and differential cross sections. These cross sections were derived from an interpolation of experimental data above $E_{\gamma}=1.2 \mathrm{GeV}$ (see Ref. [6] for details). In order to verify the above described approach [14], we compared the calculated integral cross sections for $\gamma n \rightarrow \pi^{+} \Delta^{-}$and $\gamma n \rightarrow \pi^{-} \Delta^{+}$reactions with experimental data originally published in Ref. [15] and compiled in Ref. [5]. Although the data have large uncertainties and refer to a narrow $E_{\gamma}$ region, we tried to adjust the magnitudes of integral cross sections used in Ref. [6], keeping their energy dependences. This completely phenomenological procedure eliminates obvious overestimation of the $\gamma n \rightarrow \pi^{+} \pi^{-} n$ and $\gamma n \rightarrow \pi^{0} \pi^{-} p$ experimental data and reproduces the observations made in Ref. [15] concerning the difference between the $\gamma n \rightarrow \pi^{+} \Delta^{-}$and $\gamma p \rightarrow \pi^{-} \Delta^{++}$cross sections and between the $\gamma n \rightarrow \pi^{+} \pi^{-} n$ and $\gamma p \rightarrow \pi^{+} \pi^{-} p$ ones. Fig. 3 shows the energy dependence of the total cross section of $\gamma N \rightarrow \pi \Delta$ channels. As it is seen, the modified event generator describes satisfactorily the data [5], taking into account their large uncertainties and scarcity, particularly for $\gamma n$-reactions.

The $\gamma N \rightarrow \rho N$ channel was described following the phenomenological approach of Ref.[14]. Approximations of experimental data were used above $E_{\gamma}=3 \mathrm{GeV}$ [6]. As it was stressed in Ref. [16], there is an indication of the presence of $s$-channel resonances in the subchannel $\gamma p \rightarrow \rho^{0} p$ on the level of $1 \mu \mathrm{b}$, while the non-resonant diffraction process gives the main contribution to the subchannel cross section which 


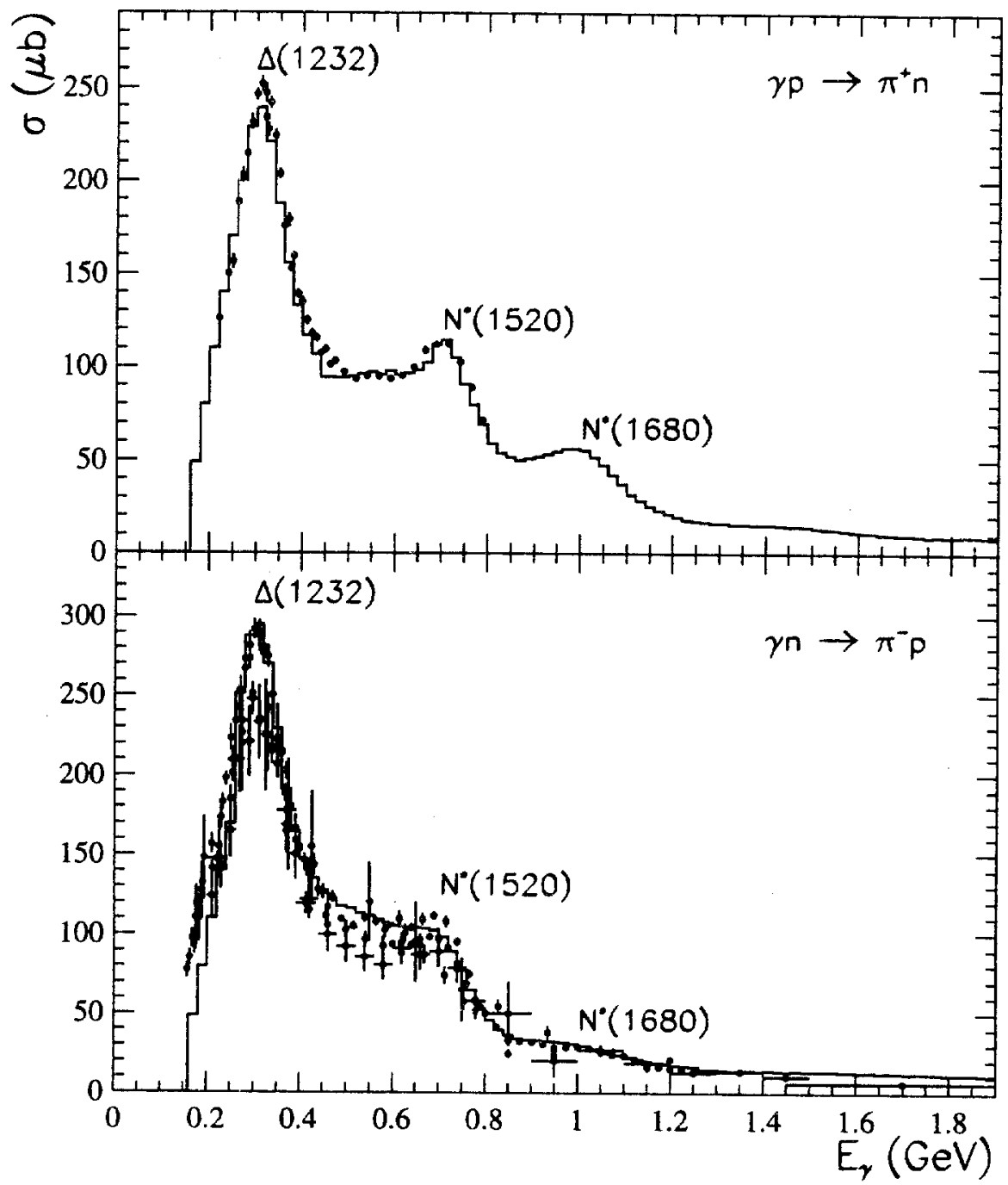

Figure 1: Energy dependence of the cross section of $\gamma p \rightarrow \pi^{+} n$ and $\gamma n \rightarrow \pi^{-} p$ reactions. Experimental points are taken from Ref. [5]. Histograms are the results of our calculation. 


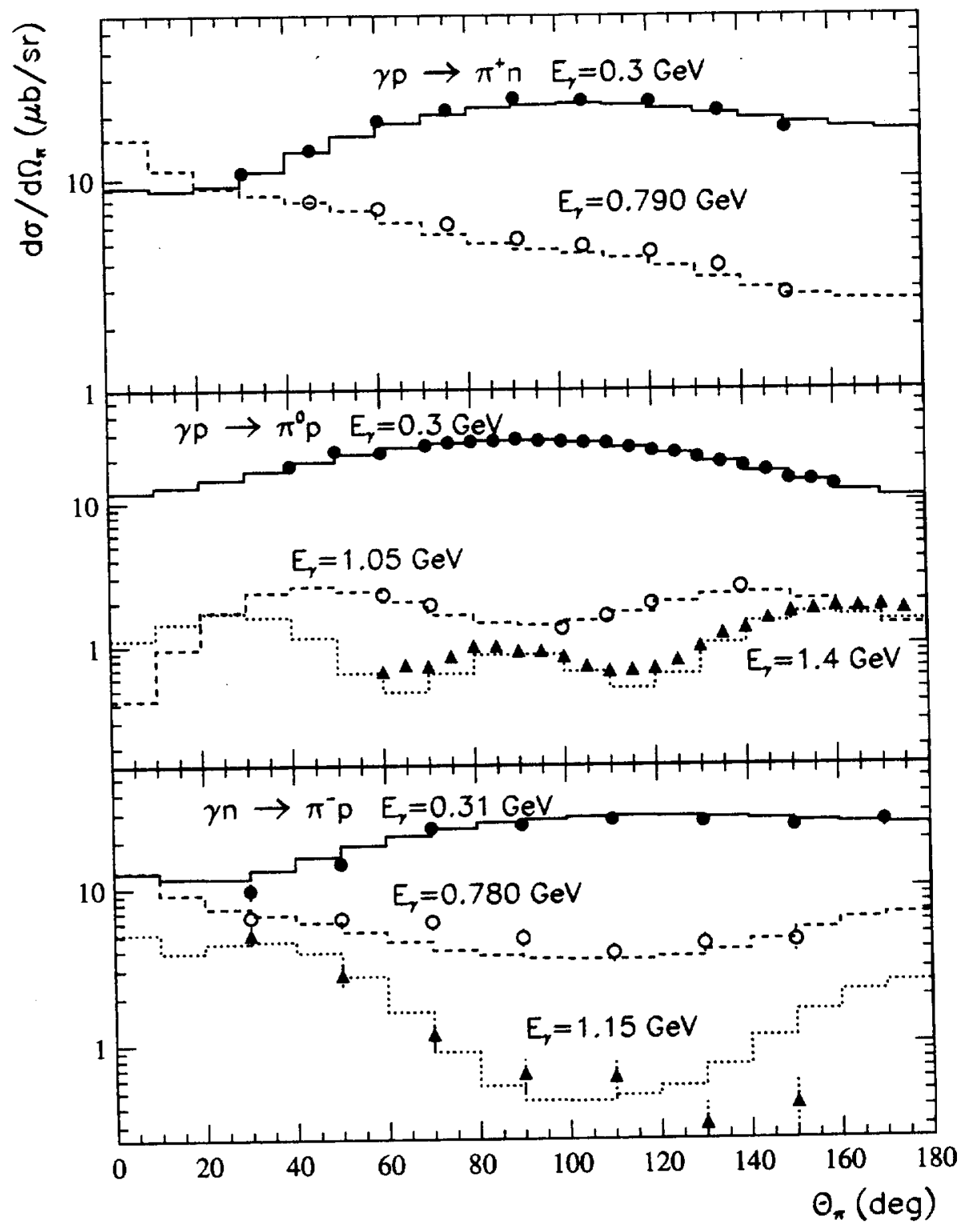

Figure 2: Angular distribution (in the c.m.system (c.m.s.)) of pions produced in $\gamma p \rightarrow \pi^{+} n, \gamma p \rightarrow \pi^{0} p$, and $\gamma n \rightarrow \pi^{-} p$ reactions at various energies of photons $E_{\gamma}$. Points were taken from Ref. [9]-[13]. Histograms are the results of our calculation. 

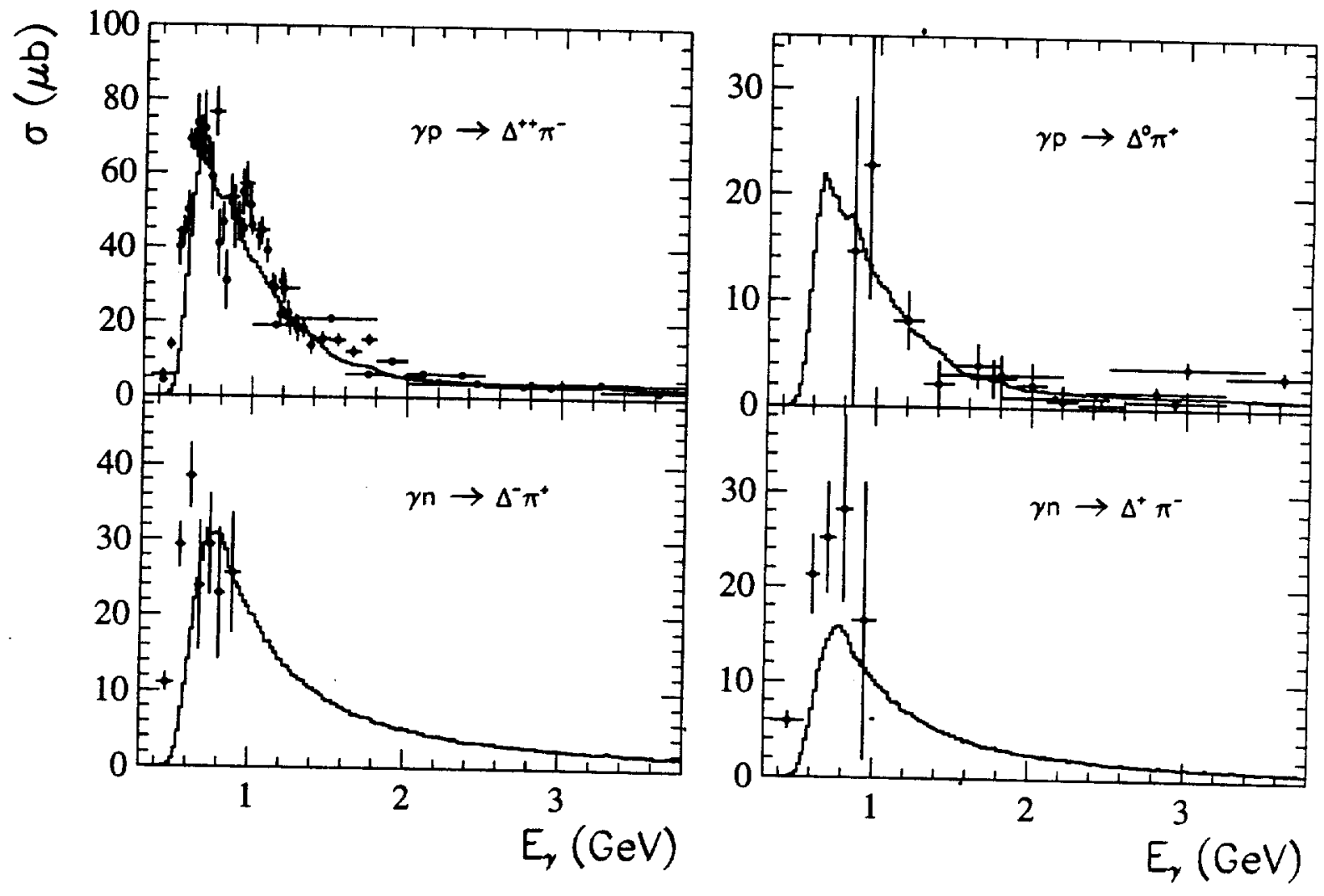

Figure 3: Energy dependence of the cross section of $\gamma p \rightarrow \pi^{-} \Delta^{++}, \gamma p \rightarrow \pi^{+} \Delta^{0}$, $\gamma n \rightarrow \pi^{+} \Delta^{-}$, and $\gamma n \rightarrow \pi^{-} \Delta^{+}$channels. Experimental points are taken from the compilation [5]. Histograms are the results of the Monte Carlo calculation with modified $\gamma N$-event generator. 
has a weak energy dependence (see Fig. 4, bottom plot). This fact leads to a prominent anisotropy in angular distributions similar to the one observed in elastic diffractive scattering of hadrons (see Fig. 5, bottom plot). There is no diffractive part in the charge-exchange subchannels $\gamma n \rightarrow \rho^{-} p$ and $\gamma p \rightarrow \rho^{+} n$ (see Ref.[16]). The s-channel resonance excitation is the dominant process in these subchannels [14]. As it is follows from experimental data [16],[18], the cross sections of $\gamma n \rightarrow \rho^{-} p$ and $\gamma p \rightarrow \rho^{+} n$ subchannels are small as compared with the $\gamma p \rightarrow \rho^{0} p$ subchannel. There are no data for the subchannel $\gamma n \rightarrow \rho^{0} n$. Therefore, as a first approximation, we assumed that a contribution of some resonance in the $s$-channel with the isospin $I_{s}$ is dominant in the subchannels $\gamma n \rightarrow \rho^{-} p, \gamma p \rightarrow \rho^{+} n$ and $\gamma n \rightarrow \rho^{0} n$. In this case the ratio $\sigma\left(\gamma n \rightarrow \rho^{-} p\right) / \sigma\left(\gamma p \rightarrow \rho^{0} p\right)$ is equal to 2 (or 0.5 ) for $I_{0}=\frac{1}{2}$ (or $\left.\frac{3}{2}\right)[16]$. The experimental value of this ratio $[16],[18]$ is about $0.1-0.4$. So, the isospin value $I_{s}=\frac{3}{2}$ was taken, and it results $\sigma\left(\gamma n \rightarrow \rho^{-} p\right)=\sigma\left(\gamma p \rightarrow \rho^{+} n\right)$, and $\sigma\left(\gamma n \rightarrow \rho^{0} n\right)=\frac{1}{2} \sigma\left(\gamma n \rightarrow \rho^{-} p\right)$.

The $\gamma p \rightarrow \eta p$ channel was calculated with the help of the isobar model of Hicks et al.[19]. This model takes into account important contributions from $S_{11}(1550)$, $S_{11}(1700)$ and $P_{11}(1750)$ states, and small admixtures of other $s$-channel resonances: $P_{13}(1860), F_{17}(1990), D_{13}(2040), G_{17}(2190)$. Due to the decisive role of $S_{11}$ states, the angular distribution of the process is not far from isotropy (see Fig. 5, top plot). As one can see from Fig. 4 (top plot), the value of $\gamma p \rightarrow \eta p$ integral cross section is negligible at $E_{\gamma}>2 \mathrm{GeV}$. To describe the $\eta$ production on neutron, we used the experimental $[21]$ ratio $\sigma(\gamma n \rightarrow \eta n) / \sigma(\gamma p \rightarrow \eta p)=\frac{2}{3}$.

The $\gamma N \rightarrow \omega N$ channel was described by empirical approximations [6] of available data. The angular distribution for $\omega$ production changes from approximate isotropy at near threshold energies $E_{\gamma}<1.4 \mathrm{GeV}$ to a sharply forward peaked one at high energies $E_{\gamma}>2 \mathrm{GeV}$ due to diffraction and pion exchange mechanisms. It was supposed that $\sigma(\gamma n \rightarrow \omega n)=\sigma(\gamma p \rightarrow \omega p)$.

An example of the quality in describing the total and differential cross sections of meson resonance photoproduction on protons is shown in Figs. 4 and 5, respectively. It should be noted here that there are not so many accurate data for these reactions, and there is no clear understanding what is the role of various mechanisms in different photon energy ranges. So, it would be reasonable to improve the description of meson resonance photoproduction when new data will appear.

\subsection{Many-body subprocesses}

Considering the multiple pion photoproduction channels $\gamma N \rightarrow i \pi N$ (with $2 \leq i \leq$ 8 ), one notes that about 80 channels are opened at $E_{\gamma} \sim 5 \mathrm{GeV}$. The complete list of $\gamma N \rightarrow i \pi N(2 \leq i \leq 8)$ channels is presented in Table $2 .^{3}$ A lot of these channels are not suitable for measurements in spark, bubble or streamer chamber experiments due to the presence of more than one neutral particle in the final state.

\footnotetext{
${ }^{3}$ As there are no systematic and detailed data on photoproduction of baryon and meson resonances in the multi-pion channels, we neglected them. So, some correlations in kinematics characteristics of pions and nucleon produced in the $\gamma N$-interaction will be lost. This is a good approximation when the main channels of $\gamma A$-interaction are analyzed, except the channel of production of resonances on nuclei [22].
} 


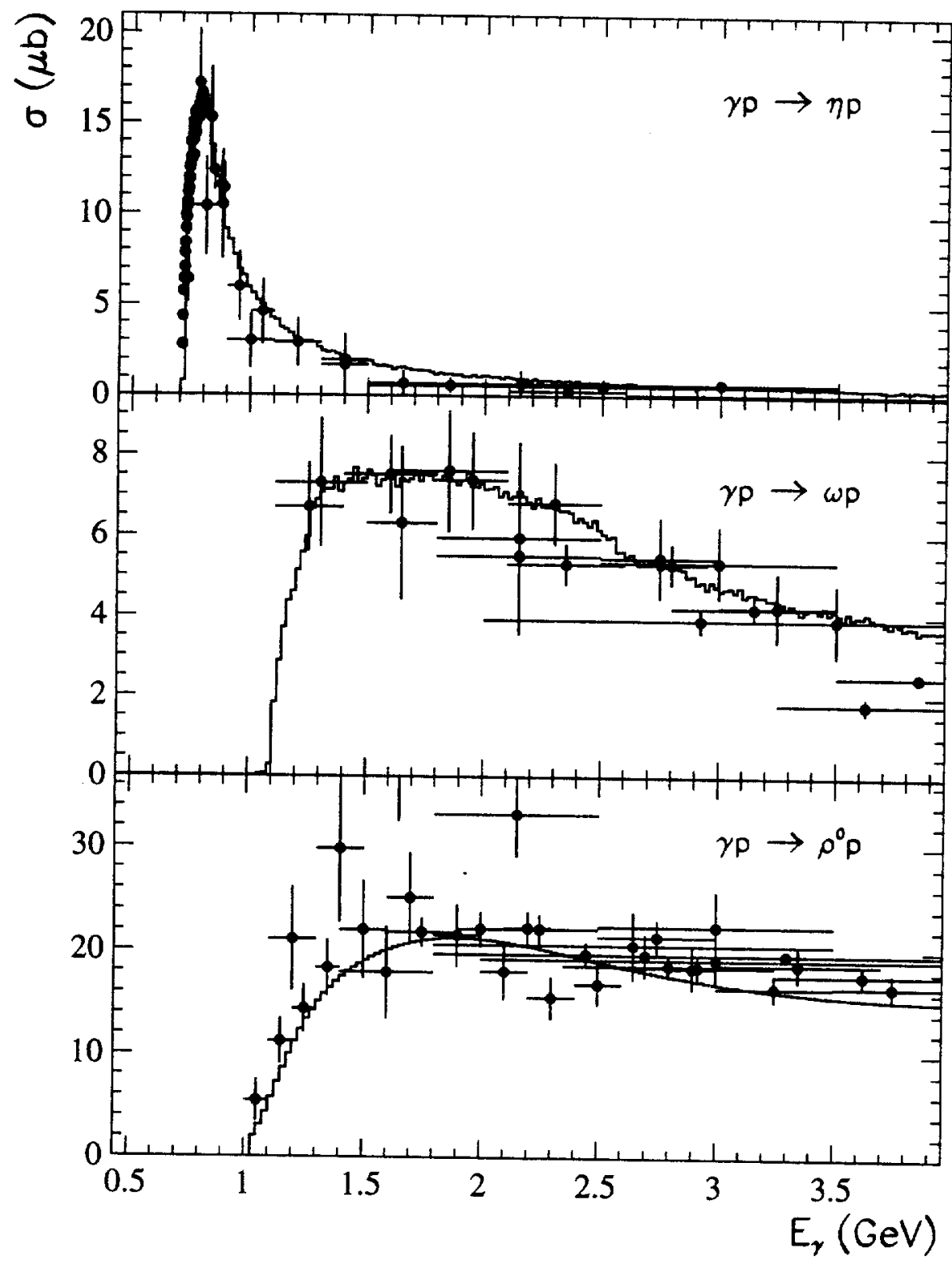

Figure 4: Energy dependence of the cross section of $\gamma p \rightarrow \eta p, \gamma p \rightarrow \omega p$, and $\gamma p \rightarrow \rho^{0} p$ channels. Experimental points are taken from compilation [5] and Ref.[17]. Histograms are the results of the Monte Carlo calculation. 


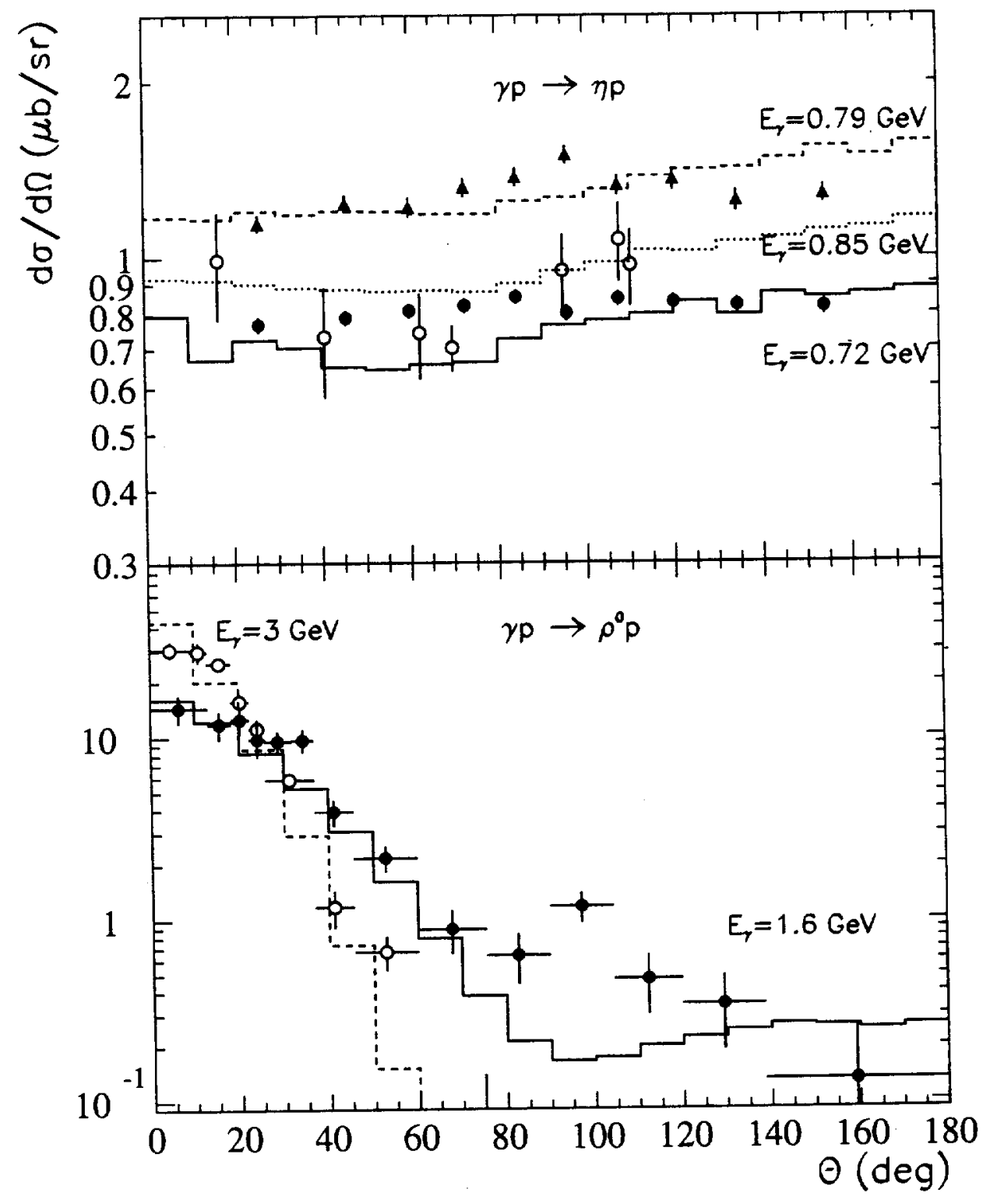

Figure 5: Angular distribution (in c.m.s.) of $\eta$ - and $\rho$-mesons produced on protons by photons with different energies $E_{\gamma}$. Points - from Refs. [17],[19],[20]. Histograms are the results of the calculations. Upper plot: solid histogram and solid circles - $E_{\gamma}=0.72 \mathrm{GeV}$, dashed histogram and solid triangles - $E_{\gamma}=0.79 \mathrm{GeV}$, dotted histogram and open circles $-E_{\gamma}=0.85 \mathrm{GeV}$. Lower plot: solid histogram and solid circles $-E_{\gamma}=1.6 \mathrm{GeV}$, dashed histogram and open circles $-E_{\gamma}=3 \mathrm{GeV}$. 
The threshold energies $E_{\gamma}^{\text {th }}$ for "measurable" channels of multiple pion production are given in Table 3 . The only possibility to reconstruct the integral cross sections of "unmeasurable" channels is to apply isotopic relations to the cross sections of channels with charged particles. To derive these relations, one has to know the reaction mechanism and the isospins of exchange and intermediate particles in the $t$ - or $s$-channels. We used such a kind of exact isotopic relations in section 2.1 for the two-body $\gamma N \rightarrow \rho N$ channels. When the number of produced pions is large and the photoproduction process is described by a sum of several diagrams, one can use statistical relations in the isospin space. The statistical approach was successfully applied in the isospin-hypercharge space [23] to consider $\pi N$ - and $p N$ interactions. In the present paper, the following semiempirical approach for multiple pion photoproduction has been adopted:

i) we found empirical approximations to calculate the integral cross sections of those channels for which data exist;

ii) we used the isospin statistical model [23] for multiple pion production, which connects unknown cross sections with measured ones, to estimate the cross section of channels for which no data are available.

The integral cross sections were evaluated by means of the expressions used in Ref. [24] for the analysis of pion production in the $N N$-interaction:

$$
\sigma(x)=\left\{\begin{array}{lll}
0 & \text { for } & E_{\gamma} \leq E_{\gamma}^{\text {th }} \\
|F(x)|^{2} & \text { for } & E_{\gamma}>E_{\gamma}^{\text {th }}
\end{array}\right.
$$

where $x=\ln \left(E_{\gamma} / E_{\gamma}^{t h}\right)$ and $F(x)$ is an effective amplitude of the process. Since the phase of $F(x)$ is not determinable, we assumed the function $F(x)$ to be real:

$$
F(x)=\sum_{n=0}^{\infty} a_{n} L_{n}^{\alpha}(x)
$$

where $a_{n}$ are the coefficients of the expansion into a series of orthonormal functions $L_{n}^{\alpha}(x)$. These functions were expressed via the generalized Laguerre polynomials $\mathcal{L}_{n}^{\alpha}(x)$ in the following way:

$$
L_{n}^{\alpha}(x)=e^{-x / 2} x^{\alpha / 2} \mathcal{L}_{n}^{\alpha}(x)
$$

and were normalized according to the expression

$$
\int_{0}^{\infty} L_{n}^{\alpha}(x) L_{m}^{\alpha}(x) d x=\frac{\Gamma(n+1+\alpha)}{\Gamma(n+1)} \delta_{n m}
$$

where $\Gamma(x)$ is the gamma-function. We used $\alpha=2$ as in Ref. [24].

The experimental data [5] on the cross section of the $\gamma N \rightarrow i \pi N$ channels were fitted by the expressions (1),(2). The values of $a_{n}$-coefficients found are given in 
Table 3. As it is seen, from two to four $a_{n}$-coefficients are sufficient for describing the experimental data with a good accuracy.

Then we found the isotopic relations between the cross sections of channels with the same number of particles in the final state but different combinations of their charge (different charge topology). To this end, we selected the statistical approach which was used by some of us in a more general form (in the isospin-hypercharge space) to describe the multiple meson production in antiproton annihilation [22]:

In the framework of this approach we deal with a system of $k$ particles in the final state $(k \geq 3)$ with isospins $j_{1}, \ldots, j_{k}$ and 3 rd projections $m_{1}, \ldots, m_{k}$, and with total isospin and 3 rd projection $J$ and $M$, respectively. The wave function of such a system $\left|\Psi_{J M}\right\rangle$, constructed from the wave functions of the component particles $\left|\Psi_{j_{1} m_{1}}>, \ldots,\right| \Psi_{j_{k} m_{k}}>$, is:

$$
\begin{aligned}
\mid \Psi_{J M}>= & \sum_{\substack{J_{1} \ldots J_{k-2} \\
\left(J_{k-2} M_{k-2} j_{k} m_{k}\right.}}\left(j_{1} m_{1} j_{2} m_{2} \mid J_{1} M_{1}\right)\left(J_{1} M_{1} j_{3} m_{3} \mid J_{2} M_{2}\right) \cdots \\
& \left(\Psi_{j_{1} m_{1}}>\left|\Psi_{j_{2} m_{2}}>\cdots\right| \Psi_{j_{k} m_{k}}>.\right.
\end{aligned}
$$

Here $J_{1}, \ldots, J_{k-2}$ and $M_{1}, \ldots, M_{k-2}$ are the intermediate isospins and 3rd projections successively composed from the isospins $j_{1}, \ldots, j_{k}$ and the 3 rd projections $m_{1}, \ldots, m_{k}$ of the particles in the final state. In the statistical approach it is supposed that there are no correlations between the wave functions of particles in the final state. Then the probability to find the system in the $J M$ state is expressed as the sum over different combinations of the $3 \mathrm{rd}$ projections (charges) of particles in the final state:

$$
\begin{aligned}
W_{J M} & \sim<\Psi_{J M} \mid \Psi_{J M}>= \\
& =\sum_{m_{1} \cdots m_{k}} \sum_{J_{1} \cdots J_{k-3}}\left(j_{1} m_{1} j_{2} m_{2} \mid J_{1} M_{1}\right)^{2}\left(J_{1} M_{1} j_{3} m_{3} \mid J_{2} M_{2}\right)^{2} \cdots\left(J_{k-2} M_{k-2} j_{k} m_{k} \mid J M\right)^{2}= \\
& =\sum_{m_{1} \cdots m_{k}} W\left(j_{1} m_{1}, j_{2} m_{2}, \cdots j_{k} m_{k} \mid J M\right) .
\end{aligned}
$$

The coefficients $W\left(j_{1} m_{1}, j_{2} m_{2}, \ldots, j_{k} m_{k} \mid J M\right)$ for the $\gamma N \rightarrow i \pi N$ reactions (with $2 \leq i \leq 8$ ) averaged over the isospins $J=1 / 2$ and $3 / 2$ are given in Table 2 . The channels whose total cross sections were available in literature, are singled out in the Table and named reference reactions. As it is seen, channels with the largest number of identical particles have the smallest probability. This is particularly evident for the channels with a large number of $\pi^{0}$-mesons.

To verify the goodness of the present approach, we used the $\gamma N \rightarrow 2 \pi N$ channel having an intermediate position between the two-body and many-body processes. Two data sets are available for the $\gamma p \rightarrow \pi^{+} \pi^{-} p$ reacton: those of Alekhin et al. [5] cover the energy region $0.5 \leq E_{\gamma} \leq 20 \mathrm{GeV}$, and those of Braghieri et al. [25], the energy region $0.5-0.8 \mathrm{GeV}$. Unfortunately, they differ from each other in the overlapping energy region. Therefore we approximated the energy dependence of the $\gamma p \rightarrow \pi^{+} \pi^{-} p$ reaction by two components: the first one describes the new data 
in the energy region $0.5 \leq E_{\gamma} \leq 0.8 \mathrm{GeV}$ while the second one describes the old data at $E_{\gamma}>0.8 \mathrm{GeV}$ (see Fig. 6). The coefficients in the expressions (1),(2) for the high-energy component are presented in Table 3. Moreover, the recent data [25] are the first measurement of the channels with neutral particles in the final state: $\gamma p \rightarrow \pi^{+} \pi^{0} n$ and $\gamma p \rightarrow \pi^{0} \pi^{0} p$. They have shown that the experimental ratio $\sigma\left(\gamma p \rightarrow \pi^{+} \pi^{0} n\right) / \sigma\left(\gamma p \rightarrow \pi^{+} \pi^{-} p\right)$ is close to the statistical estimation. We assumed a two-component energy dependence also for the $\gamma p \rightarrow \pi^{+} \pi^{0} n, \gamma n \rightarrow \pi^{+} \pi^{-} n$ and $\gamma n \rightarrow \pi^{-} \pi^{0} p$ channels. As it seen from Fig. 6, all the above mentioned channels contain contributions from the $\gamma N \rightarrow \pi \Delta$ and $\gamma N \rightarrow \rho N$ processes. Only the $\gamma p \rightarrow \pi^{+} \pi^{-} p$ channel can be unumbigiously separated into $\gamma p \rightarrow \Delta^{++} \pi^{-}, \gamma p \rightarrow \rho^{0} p$ and the so called "non-resonant" part via effective mass distribution measurements in a wide region of $E_{\gamma}$ (see Ref.[16] for details). The rest of the integral cross sections for $\gamma p \rightarrow \pi^{+} \pi^{0} n, \gamma n \rightarrow \pi^{+} \pi^{-} n$ and $\gamma n \rightarrow \pi^{-} \pi^{0} p$ channels, which can not be assigned to the $\gamma N \rightarrow \Delta \pi$ and $\gamma N \rightarrow \rho N$ channels, was attributed to the "non-resonant" part.

The statistical model overestimates the experimental ratio $\sigma\left(\gamma p \rightarrow \pi^{0} \pi^{0} p\right) /$ $\sigma\left(\gamma p \rightarrow \pi^{+} \pi^{-} p\right)[25]$. In the channel $\gamma p \rightarrow \pi^{0} \pi^{0} p$ neither the Born non-resonant term nor $\rho N$ contribution are present. Therefore this channel contains only the $\gamma p \rightarrow \pi^{0} \Delta^{+}$contribution (at least in the region $0.4 \leq E_{\gamma} \leq 0.8 \mathrm{GeV}$ ). This implies that in the equation (6) several values of intermediate isospin $J_{1}$ and 3rd projection $M_{1}$ for the $\pi^{0} \pi^{0} p$ state are suppressed, and then the statistical approach fails to give a plausible estimation for $\gamma p \rightarrow \pi^{0} \pi^{0} p$ cross section which turns out to be low [25].

\section{The $\gamma N$-event generator. Comparison between results and experimental data}

Here we describe the main features of the code used for the simulation of the $\gamma N$ interaction. For a given energy $E_{\gamma}$, each $\gamma N$-channel was sampled according to the relevant value of the integral cross section provided by the above described procedure. The kinematics of two-body channels was completely defined by extracting the emission angle of one of secondary particles according to the differential cross sections. Kinematics parameters of secondaries in multi-body channels were randomly selected according to the phase space. The channels with production of meson and baryon resonances which undergo subsequent decay were accounted for in the multi-pion channels.

We checked the event generator by comparing its results with several sets of experimental data. Concerning the $\gamma N \rightarrow 2 \pi N$ channels, experimental information on the energy dependence of the integral cross section is available for the $\gamma p \rightarrow$ $\pi^{+} \pi^{-} p$ channel defined in Table 2 as reference channel. Different contributions from both the two-body resonant channels $\gamma N \rightarrow \pi \Delta$ and $\gamma N \rightarrow \rho N$, and non-resonant part were considered. As it is shown in Fig. 6, the event generator predictions describe well the cross section data available for $\gamma N \rightarrow 2 \pi N$ channels [5],[25].

In Fig. 7 the calculated energy dependence of the cross section is compared to the data for the given exclusive channels. As it is seen, the calculation well describes the 


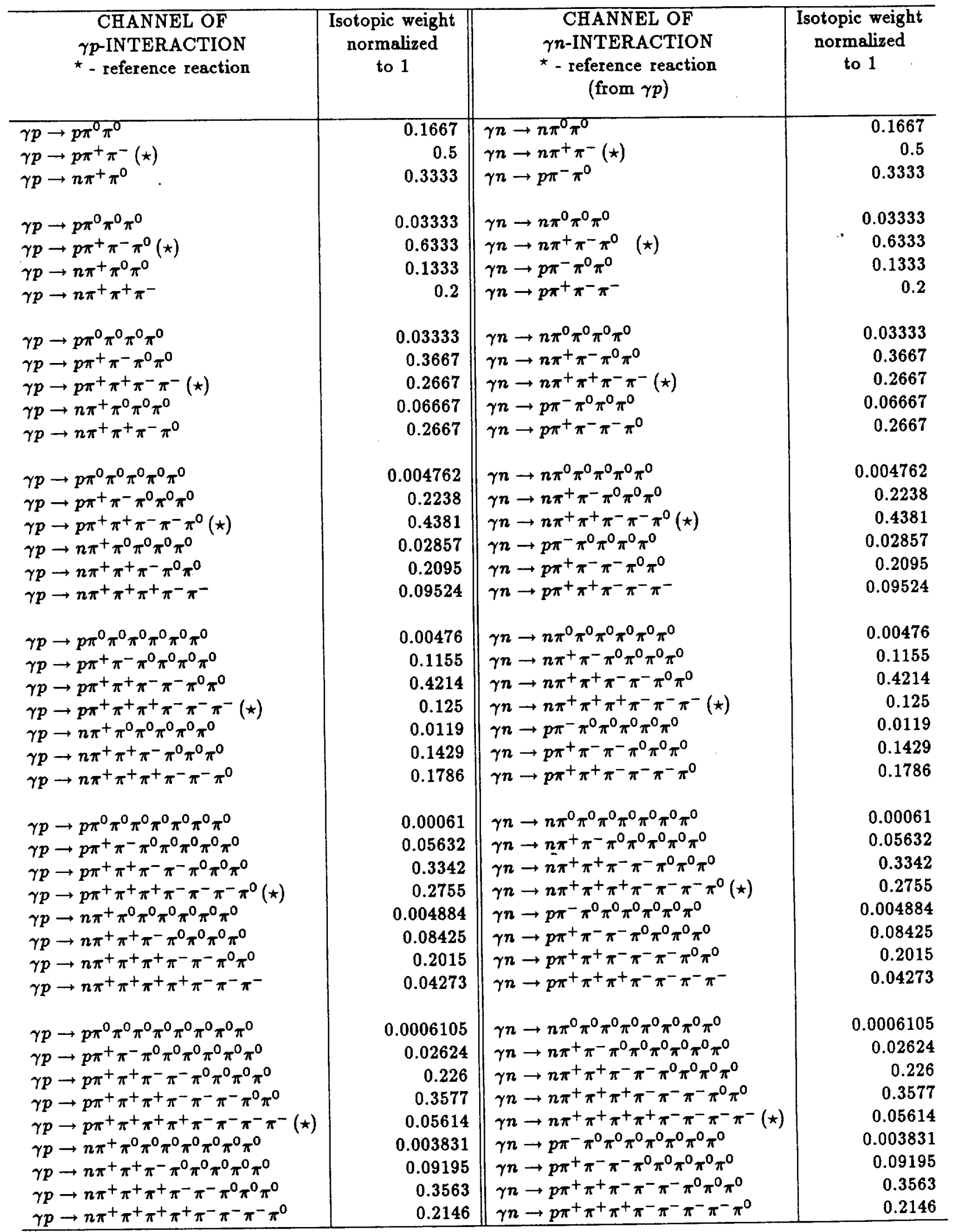

Table 2: List of $\gamma N \rightarrow i \pi N$ (with $2 \leq i \leq 8$ ) channels and the isotopic weights which connect the cross sections of unmeasured and reference channels of multiple pion production. 

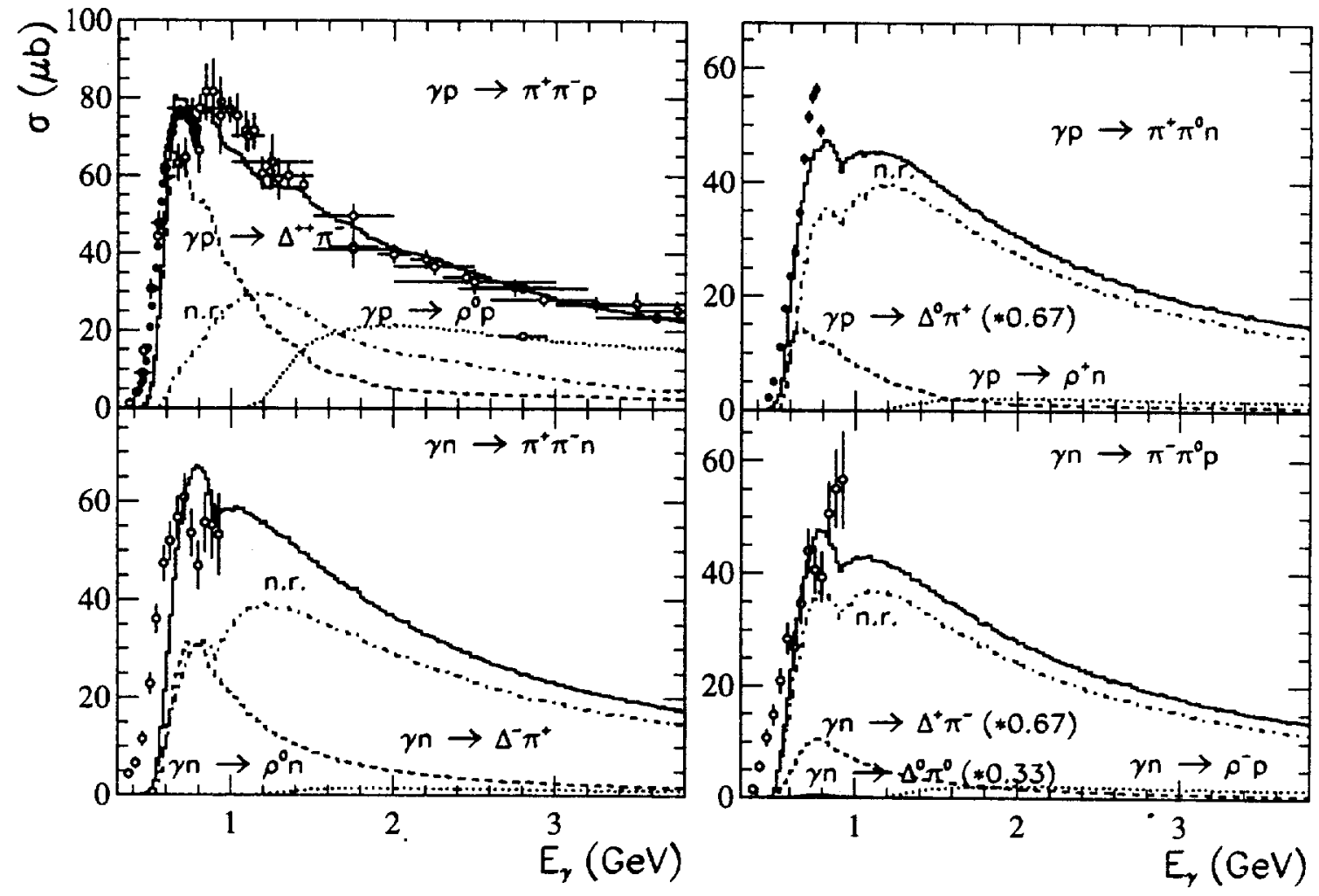

Figure 6: Energy dependence of the cross section of the $\gamma N \rightarrow 2 \pi N$ channel (solid histograms) with indication of the contribution of different subchannels: $\gamma N \rightarrow \pi \Delta$ (dashed histograms), $\gamma N \rightarrow \rho N$ (dotted histograms), and non-resonant part (dotdashed histograms). Solid circles - from Ref. [25], open circles - from Ref. [5]. 


\begin{tabular}{|l|c|c|c|c|c|}
\hline$\gamma N$ reaction channel & $E_{\gamma}^{\text {th }}$ & \multicolumn{3}{|c|}{ Coefficients of the fit between $0.10 \mathrm{GeV}$} \\
\cline { 2 - 6 } & $\mathrm{GeV}$ & $a_{0}$ & $a_{1}$ & $a_{2}$ & $a_{3}$ \\
\hline \multicolumn{5}{|c|}{$\gamma p$-interaction } \\
\hline$\gamma p \rightarrow p \pi^{+} \pi^{-}$ & 0.400 & -0.10742 & 0.63424 & 0.50893 & 0.23630 \\
$\gamma p \rightarrow p \pi^{+} \pi^{-} \pi^{0}$ & 0.506 & 0.05379 & 0.13504 & -0.03173 & \\
$\gamma p \rightarrow n \pi^{+} \pi^{+} \pi^{-}$ & 0.516 & -0.11102 & 0.24976 & -0.10591 & \\
$\gamma p \rightarrow p \pi^{+} \pi^{+} \pi^{-} \pi^{-}$ & 0.727 & -0.00098 & 0.098325 & -0.047548 & \\
$\gamma p \rightarrow p \pi^{+} \pi^{+} \pi^{-} \pi^{-} \pi^{0}$ & 0.952 & 0.20568 & -0.06299 & & \\
$\gamma p \rightarrow n \pi^{+} \pi^{+} \pi^{+} \pi^{-} \pi^{-}$ & 0.964 & 0.14433 & -0.05102 & -0.00067 & \\
$\gamma p \rightarrow p \pi^{+} \pi^{+} \pi^{+} \pi^{-} \pi^{-} \pi^{-}$ & 1.215 & 0.06190 & -0.01921 & & \\
$\gamma p \rightarrow p \pi^{+} \pi^{+} \pi^{+} \pi^{-} \pi^{-} \pi^{-} \pi^{0}$ & 1.481 & 0.11137 & -0.04094 & & \\
$\gamma p \rightarrow n \pi^{+} \pi^{+} \pi^{+} \pi^{+} \pi^{-} \pi^{-} \pi^{-}$ & 1.495 & 0.01684 & -0.00054 & \\
$\gamma p \rightarrow p \pi^{+} \pi^{+} \pi^{+} \pi^{+} \pi^{-} \pi^{-} \pi^{-} \pi^{-}$ & 1.788 & 0.033678 & -0.013025 & \\
\hline \multicolumn{7}{|c|}{$\gamma n$-interaction } \\
$\gamma n \rightarrow n \pi^{+} \pi^{-}$ & 0.322 & 0.19699 & 0.10912 & & \\
$\gamma n \rightarrow p \pi^{-} \pi^{0}$ & 0.313 & 0.10577 & 0.21707 & -0.06863 & 0.00023 \\
$\gamma n \rightarrow p \pi^{+} \pi^{-} \pi^{-}$ & 0.512 & 0.07522 & 0.017378 & & \\
$\gamma n \rightarrow n \pi^{+} \pi^{+} \pi^{-} \pi^{-}$ & 0.728 & 0.08950 & 0.02417 & & \\
$\gamma n \rightarrow p \pi^{+} \pi^{-} \pi^{-} \pi^{0}$ & 0.716 & 0.08950 & 0.02417 & \\
\hline
\end{tabular}

Table 3: Values of the threshold energies $E_{\gamma}$ and of the coefficients of the polynomial fit to the integral cross section of $\gamma N \rightarrow i \pi N$ reactions $(2 \leq i \leq 8)$.

reference multi-body channels $\left(\gamma p \rightarrow 2 \pi^{+} 2 \pi^{-} p, 2 \pi^{+} 2 \pi^{-} \pi^{0} p, 3 \pi^{+} 3 \pi^{-} p, 3 \pi^{+} 3 \pi^{-} \pi^{0} p\right)$, and the $3 \pi^{+} 2 \pi^{-} n$ channel whose cross section was found using the statistical model.

In Fig. 8 the phenomenological approach is compared to the total cross section of hadron production in the $\gamma p$-interaction. As it is seen in the upper plot, the approach accurately describes the data and reasonably well estimates the distribution in multiplicity of produced charged particles and the energy dependence of the cross sections for production of the given number of charged particles. It should be noted that the method overestimates by $20-50 \%$ the cross section of production of one charged particle which includes contributions from channels with multiple $\pi^{0}$ production like $\gamma p \rightarrow p \pi^{0} \pi^{0}, p \pi^{0} \pi^{0} \pi^{0}, n \pi^{+} \pi^{0} \pi^{0}$, and so on. In the lower plot our approach is compared to the results of the generator by Corvisiero et al. [6], and are shown the contributions to the total hadronic cross section from the channels with production of a given number of pions. As it is seen, the contribution from the two pion production channels and the summed contribution of all other multiple pion ( 3 to $8 \pi$ ) production channels are comparable in the energy region $E_{\gamma} \geq 1.5-2 \mathrm{GeV}$. This stress the need for the inclusion of multi-pion channels for a good description of the photoproduction processes up to high energies. This is the reason why the $\gamma N$-event generator of Corvisiero et. al. [6] which does not take into account all multi-pion channels can not describe correctly even the total cross section of hadron production (see Fig. 8).

Finally, in Figs. 9 and 10 it is shown how well this phenomenological method 


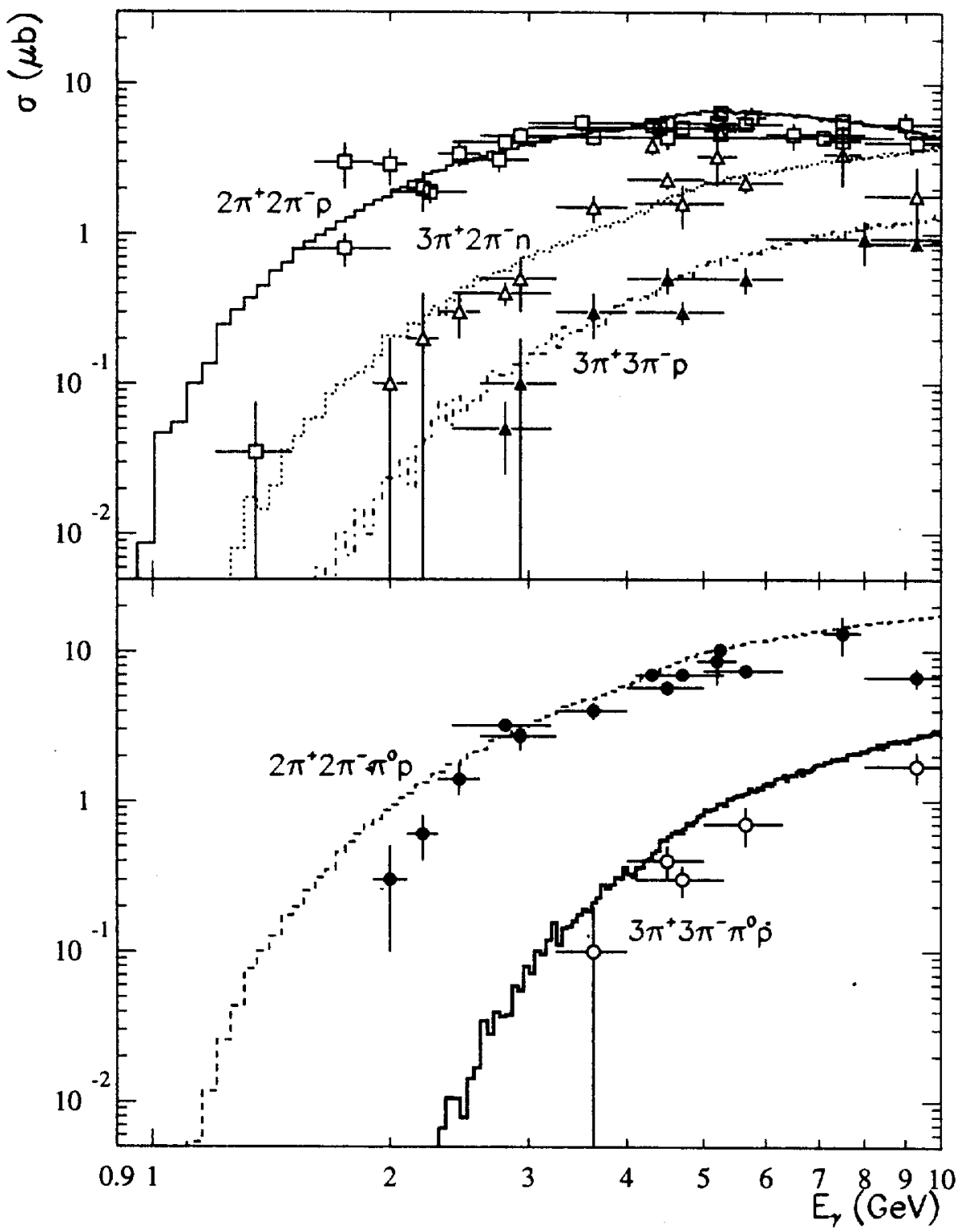

Figure 7: Comparison between the measured energy dependence of the cross section of the exclusive channels of $\gamma p$-interaction and the Monte Carlo simulation. Points - from Ref.[5], histograms - calculated results: $2 \pi^{+} 2 \pi^{-} p$ channel - open squares and solid line, $2 \pi^{+} 2 \pi^{-} \pi^{0} p$ - full circles and dashed line, $3 \pi^{+} 2 \pi^{-} n$ - open triangles and dotted line, $3 \pi^{+} 3 \pi^{-} p$ - full triangles and dash-dotted line, $3 \pi^{+} 3 \pi^{-} \pi^{0} p$ - thick solid line and open circles. 


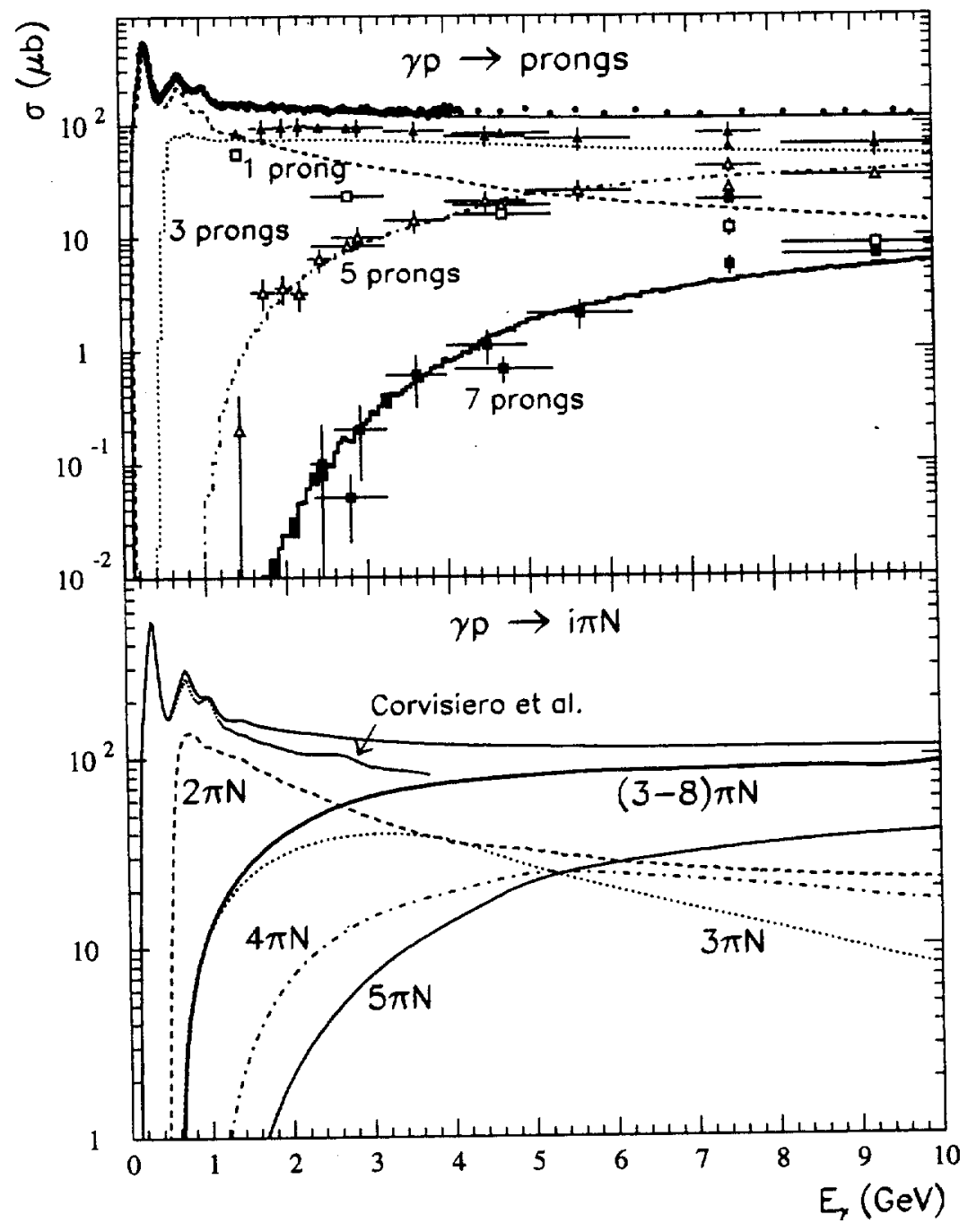

Figure 8: Energy dependence of the cross sections of charged particle production (upper plot) and multiple pion production (lower plot) in $\gamma p$-interaction. Experimental data [5] and calculation results for the cross sections are presented respectively, by open squares and dashed histogram for 1 prong, by full triangles and dotted histogram for 3 prongs, by open triangles and dash-dotted histogram for 5 prongs and by full squares and thick solid line histogram for 7 prongs. Prediction for $2 \pi$ contribution - dashed line and for $(3-8) \pi$ production - thick solid line. $3 \pi, 4 \pi$ and $5 \pi$ contributions - dotted, dash-dotted and solid lines, respectively. The total hadron photoproduction cross section is presented by experimental points ([26-28], circles) and solid line (our generator). Condensed dotted line - the sum of cross sections of hadron production channels in the generator of Corvisiero et al. [6]. 
describes the momentum and angular distributions of pions produced in the $\gamma p$ interaction originated by bremsstrahlung photons of maximum energy $E_{\gamma}^{\max }=6$ $\mathrm{GeV}[29]$.

In conclusion, the good description of a broad range of experimental data on $\gamma N$ interaction at energies up to $10 \mathrm{GeV}$ validate the use of the Monte Carlo $\gamma N$-event generator inside the INC model for studying photonuclear reactions.

\section{The $\gamma A$-event generator}

As done by Gudima et al. in Ref. [1], we have described the two-nucleon absorption cross section of a photon on a nucleus, $\sigma_{A}$, with the help of the quasideuteron model as:

$$
\sigma_{A}=k Z(1-Z / A) \sigma_{d}
$$

where $\sigma_{d}$ is the cross section for deuteron photodisintegration $\gamma d \rightarrow n p$ derived from the compilations in Ref. [30], $A$ and $Z$ are the mass and charge numbers of the relevant nucleus, respectively, and $k \approx 10$ [1] is an empirical constant. To calculate the angular distribution of nucleons in the reaction $\gamma d \rightarrow n p$, we have used the empirical approximation given in [1]. Despite the cross section $\sigma_{d}$ decreases strongly with the photon energy, the two-nucleon absorption mechanism noticeably competes with the single-nucleon absorption mechanism up to $E_{\gamma} \sim 0.5 \mathrm{GeV} .^{4}$

\subsection{Elementary hadron-nucleon interactions}

The fast hadrons produced in a primary $\gamma N$-interaction of the single-nucleon absorption mechanism initiate a cascade of successive quasi-free hadron-nucleon collisions inside the nucleus. As the $\Delta$-isobar and $\rho$-meson have large widths, their decay mean free path is comparable to the internucleonic distance. Then for a sake of simplicity we assumed that $\Delta$ and $\rho$ decay in $\pi \mathrm{N}$ and $2 \pi$, respectively, before interacting with an intranuclear nucleon. The $\omega$-meson has a much smaller width, so that it may either interact with a nucleon or decay inside the nucleus. Finally, we considered the $\eta$-meson as a stable particle since its width is very small. Then, in the INC model we took into account the following elementary processes:

$$
\begin{gathered}
\pi N \rightarrow \pi N, \pi(N N) \rightarrow N N, \pi N \rightarrow \pi \pi N ; \pi N \rightarrow(i+1) \pi N, \\
\eta N \rightarrow \eta N, \eta N \rightleftharpoons \pi N, \eta N \rightarrow \pi \pi N, \eta(N N) \rightarrow N N, \eta(N N) \rightarrow \pi N N, \\
\omega N \rightarrow \omega N, \omega N \rightleftharpoons \pi N, \omega N \rightarrow \pi \pi N, \omega(N N) \rightarrow N N, \omega(N N) \rightarrow \pi N N, \\
N N \rightarrow N N, N N \rightarrow \pi N N, N N \rightarrow i \pi N N(i \geq 2) .
\end{gathered}
$$

The empirical approximations for the integral and differential cross sections of $N N$ and $\pi N$-interactions and the phenomenological estimations [22] for the total and partial cross sections of $\eta N$ - and $\omega N$-interactions were used in the calculation.

\footnotetext{
${ }^{4}$ This competition may be much severe if the contribution of channels with pion production $\gamma+(n p) \rightarrow i \pi N N(i \geq 1)$ is taken into account.
} 

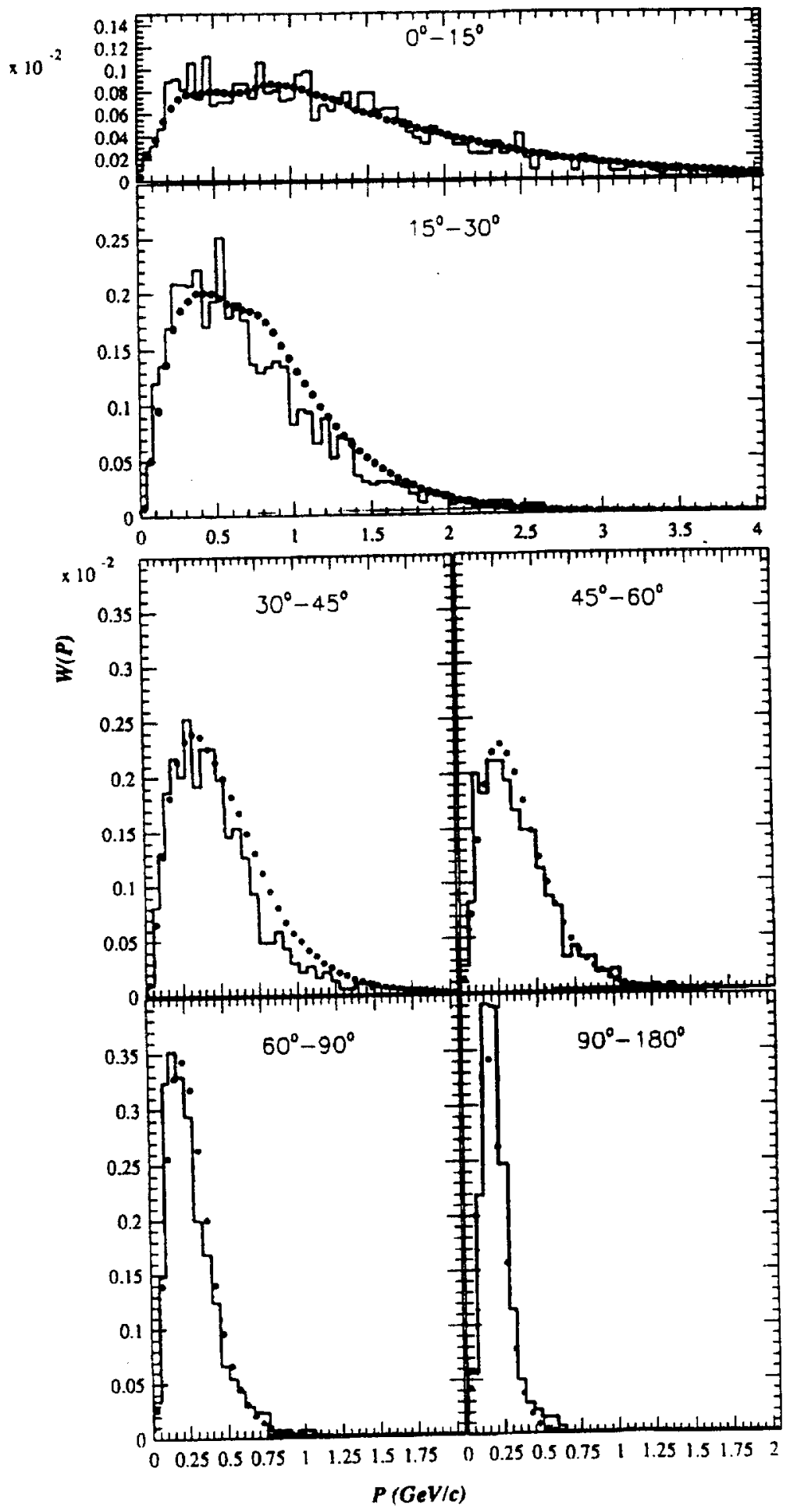

Figure 9: Momentum spectrum (in arb. units) of $\pi^{-}$mesons produced on protons by bremsstrahlung photons with a maximum energy $E_{\gamma}^{\max }=6 \mathrm{GeV}$ at different angle intervals in the lab. system. Histograms - data from Ref. [29]; points - calculated results. 


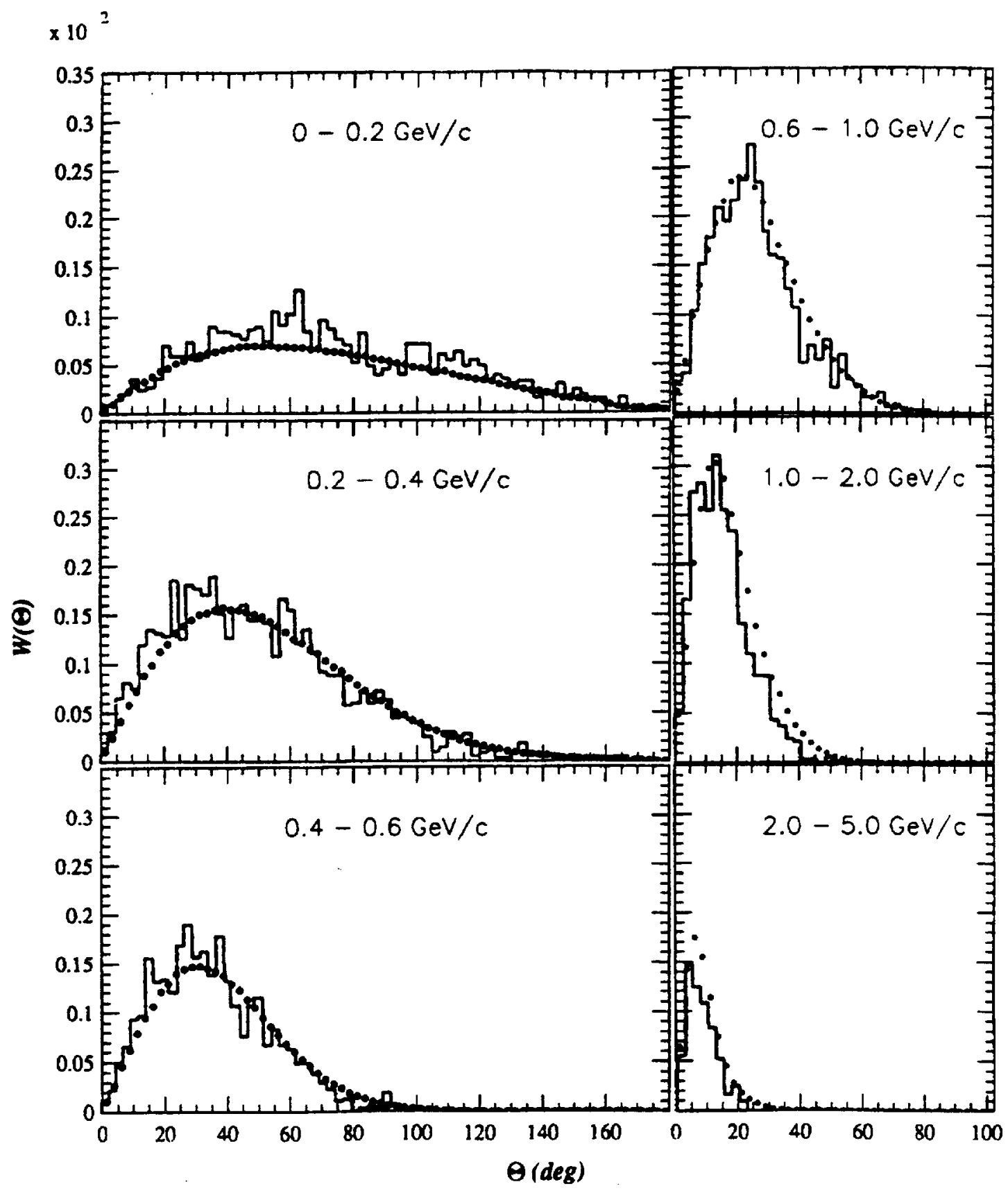

Figure 10: Angular distribution (in arb. units) of $\pi^{-}$produced on protons by bremsstrahlung photons with a maximum energy $E_{\gamma}^{\max }=6 \mathrm{GeV}$ for the given intervals of $\pi^{-}$momentum in the lab. system. Notations - as in Fig. 9. 
The two-nucleon absorption of $\pi-; \eta$ - and $\omega$-mesons was described in the framework of the quasideuteron model [22]. As in the case of $\gamma N$-interaction, the calculation of the hadron-nucleon interactions was performed with the help of a Monte Carlo event generator code.

\subsection{Calculation of the intranuclear cascade}

The INC model is a numerical method for the solution of a kinetic equation with a many-body distribution function which describes hadron transport in a nucleus. One needs to use the many-body distribution functions when the exclusive characteristics of a process must be calculated, including properties of the residual nucleus. Calculations of intranuclear cascades were performed by using Monte Carlo technique. Foundation of INC model and details of the calculation are given in Ref. [31]. Here we limit to notice that a specific feature of a photonuclear reaction at high energies is the production of a multi-pion system inside a nucleus. This makes necessary to take into account the knock out of the intranuclear nucleons by the fast pionic component of INC, so that the slower nucleons pass through a lower density nuclear region undergoing less rescattering (so called trawling effect). Therefore we used the version of INC model which takes into account the local depletion of nuclear density during the development of the cascade (see Ref. [31] for details).

An excited residual nucleus is formed after the completion of the intranuclear cascade. The INC model describes in detail the dissipation of the photon energy into the nucleus showing that the residual nuclei have wide distributions of excitation energy, $E^{\star}$, momentum, $\mathrm{P}$, angular momentum, $\mathrm{I}$, and nucleon, $\mathrm{A}$, and proton, $\mathrm{Z}$, numbers. The decay mechanism of the residual nucleus is determined by its excitation energy $\mathrm{E}^{\star}$. For $\mathrm{E}^{\star} \leq 2 \mathrm{MeV} /$ nucleon, the main mechanism is the evaporation, that is the successive emission of particles from the residual nucleus, or its fission. When the excitation energy of the nucleus gets close to its total binding energy ( $\mathrm{E}^{\star} \geq 5 \mathrm{MeV} /$ nucleon), the explosive multifragment decay mechanism dominates. In the region of intermediate excitation energies $\left(2 \leq E^{\star} \leq 5 \mathrm{MeV} /\right.$ nucleon $)$ both decay mechanisms coexist.

The calculation of the de-excitation of the residual nucleus was performed by using Monte Carlo method. The de-excitation of the moderately excited nucleus was described by the evaporation statistical model which takes into account the thermal damping of shell effects in nuclei [32]. Fission was described in the framework of the diffusion model; the evaporation from excited fission fragment was also taken into account [33]. The disintegration of the hot nucleus was described by means of the statistical model of multifragmentation [34].

\section{Comparison between $\gamma \mathrm{A}$-generator results and photonuclear data}

In the previous papers [1] the INC model was applied to describe measured energy spectra and angular distributions of protons, cross section of neutron emission pro- 
cesses, isotope yield, and fission cross section in $\gamma A$-interactions. Since the largest amount of the data were obtained with bremsstrahlung photons, it was not possible to test the model in detail. In the recent years, new photonuclear data, including also $\pi$ - and $\eta$-meson spectra, have been obtained with quasi-monochromatic photons. Though these data lie in the energy region $E_{\gamma} \leq 1 \mathrm{GeV}$, they provide a good test for this extended version of the INC model, considering that the description of $\gamma N \rightarrow \pi N$ and $\gamma N \rightarrow 2 \pi N$ subprocesses, which play the major role in this region, was deeply modified, and that the $\gamma N \rightarrow \eta N$ subprocess was taken into account for the first time in the present paper.

\subsection{Meson production}

Different kinematic regions in pion photoproduction have been studied through systematic measurements of the double differential cross section. The high energy part of the pion spectrum has been investigated by Baba et al.[35] with photons of energies $0.3 \leq E_{\gamma} \leq 0.8 \mathrm{GeV}$ detecting pions emitted at $\theta_{\pi} \leq 45^{\circ}$. The lower energy part of the pion spectrum has been measured by Arends et al.[3] with photons of energies $0.2 \leq E_{\gamma} \leq 0.4 \mathrm{GeV}$ at pion angles $\theta_{\pi}>45^{\circ}$.

Fig. 11 shows the evolution in shape of the pion spectrum with photon energy, at forward angle, $\theta_{\pi}=44^{\circ}$, together with the contributions of the different elementary processes for the cases when the single pion production dominates and when the double pion production is noticeable. At $E_{\gamma} \sim 0.8 \mathrm{GeV}$, three maxima are predicted in the spectrum: the high-energy maximum is caused by the quasi-free production of one pion, the two others by the $\gamma N \rightarrow 2 \pi N$ channel and by the final state interaction of produced pions. The contribution from the process with charge exchange is small. At this photon energy, both the $\gamma N \rightarrow \pi \Delta$ subchannel and non-resonant subchannel contribute to the $\gamma N \rightarrow 2 \pi N$ channel (see Fig. 6). These subchannels give comparable contributions to the inclusive spectrum of pions produced on a nucleus. At $E_{\gamma} \sim 0.6 \mathrm{GeV}$, the pion inclusive spectrum at $P_{\pi}<0.4 \mathrm{GeV} / \mathrm{c}$ is also formed by the products of the $\gamma N \rightarrow 2 \pi N$ channel. But the contributions from the quasi-free double pion production and final state interaction overlap now, so that there are two maxima in the spectrum. At low photon energy, $E_{\gamma} \sim 0.3 \mathrm{GeV}$, the pion spectrum has a maximum caused by the quasi-free production of a single pion and a low-energy shoulder caused by the final state interaction.

The evolution in shape of the pion spectrum with the pion angle at low primary energy $E_{\gamma} \sim 0.4 \mathrm{GeV}$ is shown in Fig. 12. At large angle, $\theta_{\pi}=108^{\circ}$, the contribution from the quasi-free production of one pion is strongly suppressed, and the pions which undergo the final state interaction form the spectrum with a single maximum at $\mathrm{P}_{\pi} \sim 0.15 \mathrm{GeV} / \mathrm{c}$. At small angles, $\theta_{\pi}=28^{\circ}$ and $48^{\circ}$, the spectrum has two maxima caused by the quasi-free production of a pion (at high $P_{\pi} \sim 0.3 \mathrm{GeV} / \mathrm{c}$ ) and by the final state interaction (at low $P_{\boldsymbol{\pi}} \sim 0.1 \mathrm{GeV} / \mathrm{c}$ ).

Thus, the spectra evolve to the shape with a single maximum both with decreasing the photon energy (see Fig. 11), and with increasing the pion angle (see Fig. 12).

The position of the peak in the quasi-free pion production cross section is sen- 


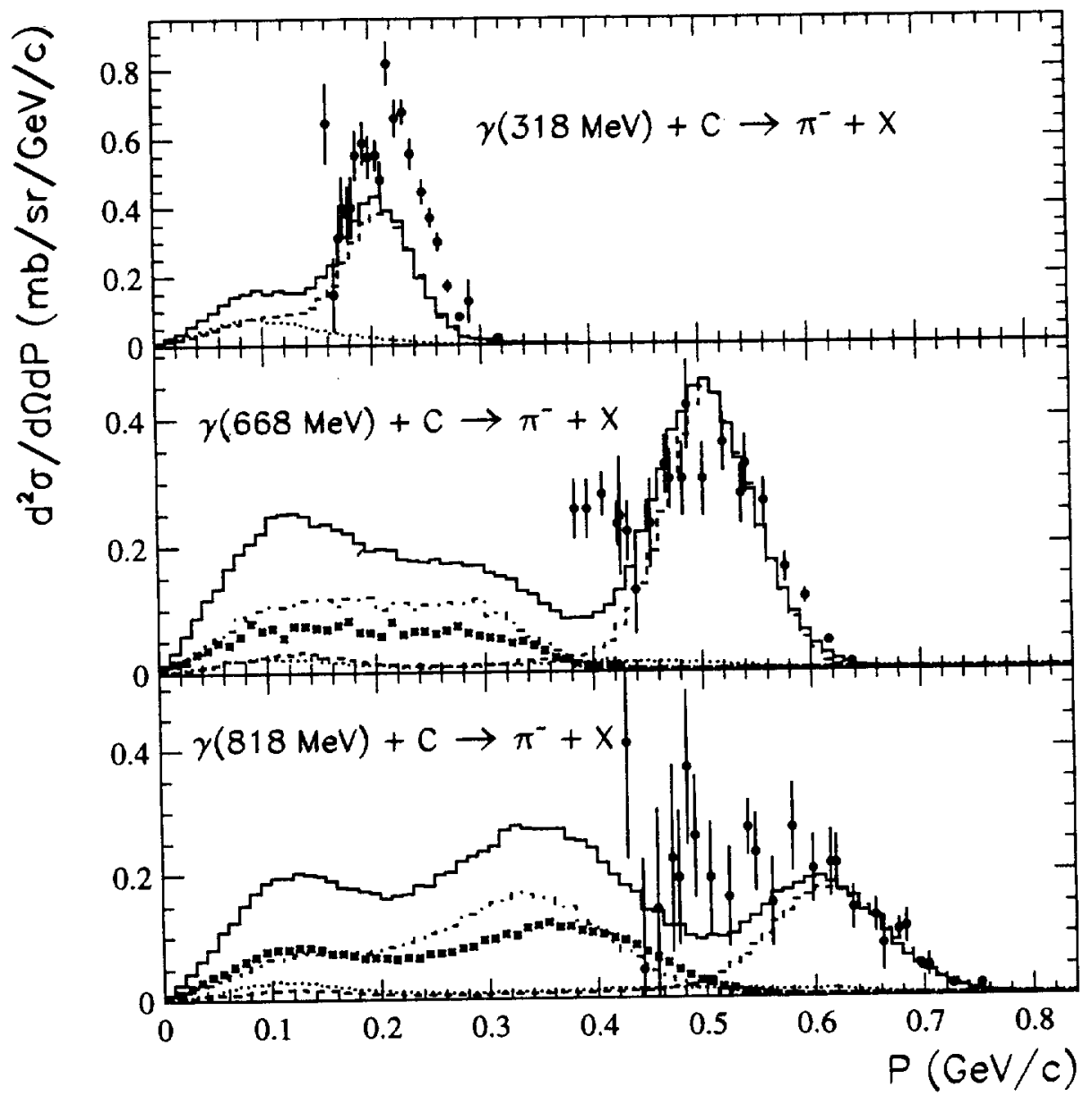

Figure 11: Double differential cross section of $\pi^{-}$production on carbon by photons with different energies at angle $\theta_{\pi}=44^{\circ}$ in the lab. system. The data are from Ref. [35]; the histograms are the Monte Carlo results. Contribution from pions produced in $\gamma n \rightarrow \pi^{-} p$ subprocess and emitted without rescattering or after elastic scattering is shown by the dashed histogram. Contribution from $\pi^{0}$ photoproduction in $\gamma p \rightarrow \pi^{0} p$ and $\gamma n \rightarrow \pi^{0} n$ subprocesses with subsequent charge exchange $\pi^{0} n \rightarrow$ $\pi^{-} p$ is shown by the dotted histogram. Contributions from $\gamma N \rightarrow \pi \Delta$ and nonresonant $\gamma N \rightarrow 2 \pi N$ subchannels with subsequent $\pi N$-interactions in the final state in each one are shown by the dot-dashed histogram and solid squares, respectively. 


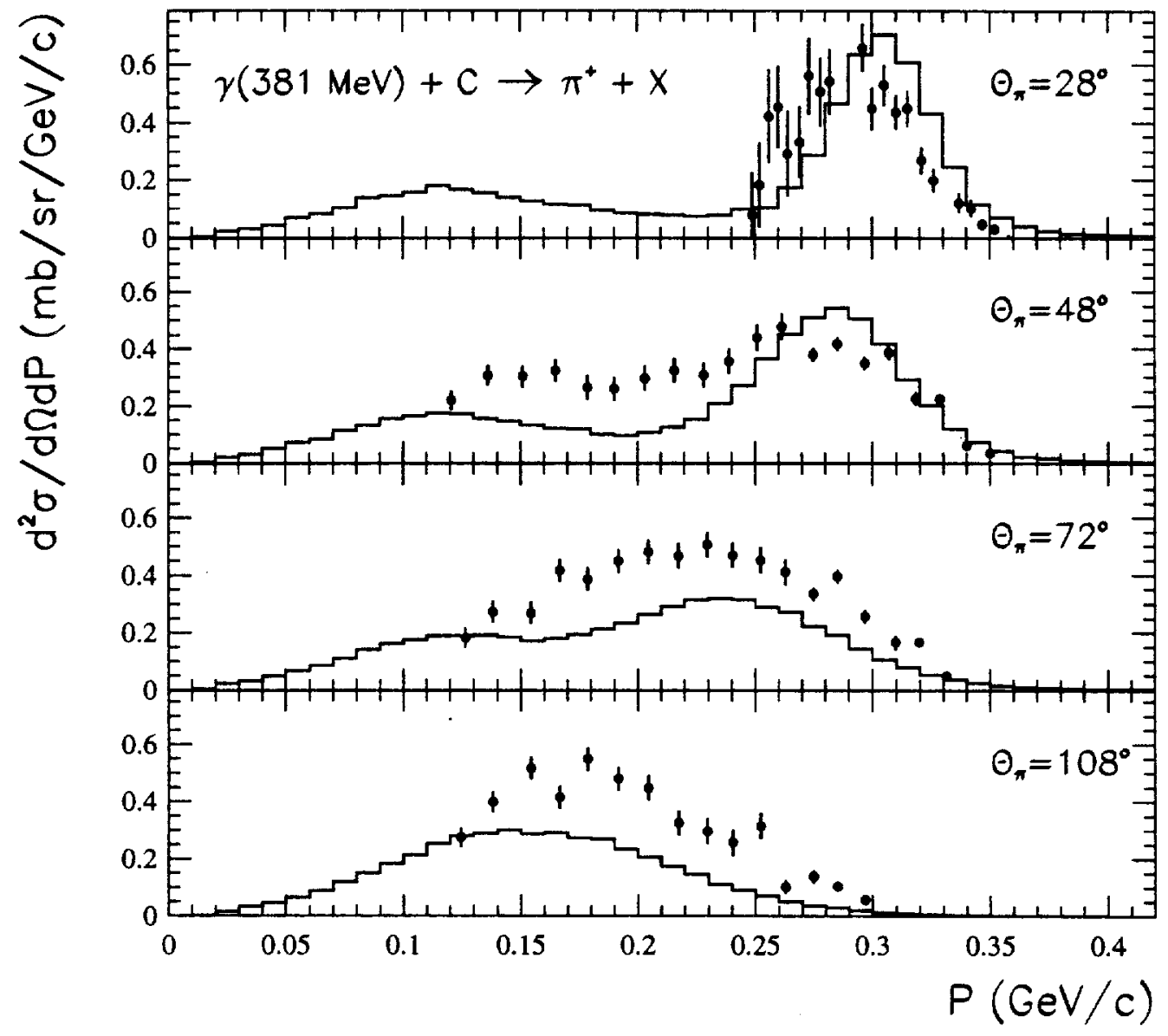

Figure 12: Double differential cross section of $\pi^{+}$produced on carbon by photons with energy $E_{\gamma}=0.38 \mathrm{GeV}$ at different angles $\theta_{\pi}$ in lab. system. The data are from Refs [3], [35]; the histograms are the Monte Carlo results. 
sitive to the value of the pion potential well, $\mathrm{V}_{x}$. Iljinov et al.[36] performed a quasi-classical calculation with the second order pion-nucleus optical potential and showed that $\mathrm{V}_{\pi}$ has a radial dependence, and that with the value $\mathrm{V}_{\pi} \approx 0$ one can describe the pion production which take place at the nuclear surface $(\pi \mathrm{A}$ - and $\bar{p} \mathrm{~A}$ reactions). On the contrary, pion photoproduction is uniformly distributed over the nuclear volume, and then the relevant data are better described by the calculation with $\mathrm{V}_{\pi}=-35 \mathrm{MeV}$ as it is shown in Fig. 13. This is also a consequence of the radial dependence of pion-nucleus potential well.

Moreover, the INC model well describes the shift of the peak in position, $\mathrm{P}_{f}$ $\mathrm{P}_{0}$, in the momentum spectrum of the quasi-free production of one pion on a nucleus with respect to its position in the pion production on a free nucleon (see Fig. 14, top plot), the width $2 \Gamma$ and height $(\mathrm{d} \sigma / \mathrm{d} \Omega)$ of the quasi-free peak in the energy range $0.3 \leq E_{\gamma} \leq 0.8 \mathrm{GeV}$ (see Fig. 14, center and bottom plots, respectively).

Fig. 15 (upper plot) shows the energy dependence of the double differential cross section for photoproduction of relatively low-energy $\left(0.1 \leq \mathrm{P}_{\pi} \leq 0.2 \mathrm{GeV} / \mathrm{c}\right)$ pions on carbon at $\theta_{\pi}=90^{\circ}$, measured by Goncharov et al. [37]. Multiple pion production channels have been investigated due to these kinematical conditions (see the discussion of Figs.11 and 12) at $E_{\gamma} \geq 0.6 \mathrm{GeV}$. They are the only available data to check the model of multiple pion photoproduction on nuclei. Fig. 15 (lower plot) shows the energy dependence of the total cross section of $\pi^{+}, \pi^{-}$and $\pi^{0}$ production on carbon. Both energy dependences in Fig. 15 have a maximum caused by the $\Delta_{33}$-resonance, and a high-energy $\left(E_{\gamma} \geq 0.6 \mathrm{GeV}\right)$ part which is smoothly energy dependent. The cross section of $\pi^{+}$and $\pi^{-}$production are approximately equal over the examined energy range. The cross section of $\pi^{0}$-production is about a factor of two larger than the cross section of $\pi^{+}$or $\pi^{-}$production at the $\Delta$-resonance peak, but becomes equal to the others at energy $E_{\gamma} \geq 0.6 \mathrm{GeV}$. It is seen from the Figure that, taking into account the large uncertainties in the data, the model agrees rather well with experiments [37]-[39].

The total cross section of pion photoproduction as a function of the nucleon number $A$ is shown in Fig. 16. The $A$-dependence can be parameterized by the expression $\sigma_{\pi}(A) \sim A^{\alpha}$, where $\alpha$ is weakly dependent on the energy $E_{\gamma}$, and equal to 0.65 . Such value of $\alpha$ originates from the volume production and the strong contribution of the final state interaction of pions (charge exchange and absorption by $N N$-pairs in a nucleus).

Finally, in Figs. 17 and 18 we show how well the INC model describes the new data of Röbig-Landau et al. [40] on the $\eta$ photoproduction on nuclei near the threshold energies.

\subsection{Proton emission}

Systematic measurements of the double differential cross section of proton photoemission are given in Refs. [41]-[44]. Fig. 19 shows the inclusive proton spectra at the given energies $E_{\gamma}$ and emission angles $\theta_{p}$. The main features of the inclusive proton spectrum from photon interaction with light nuclei are determined by the emission mechanisms. If a proton is emitted as a recoil nucleon from the quasi- 


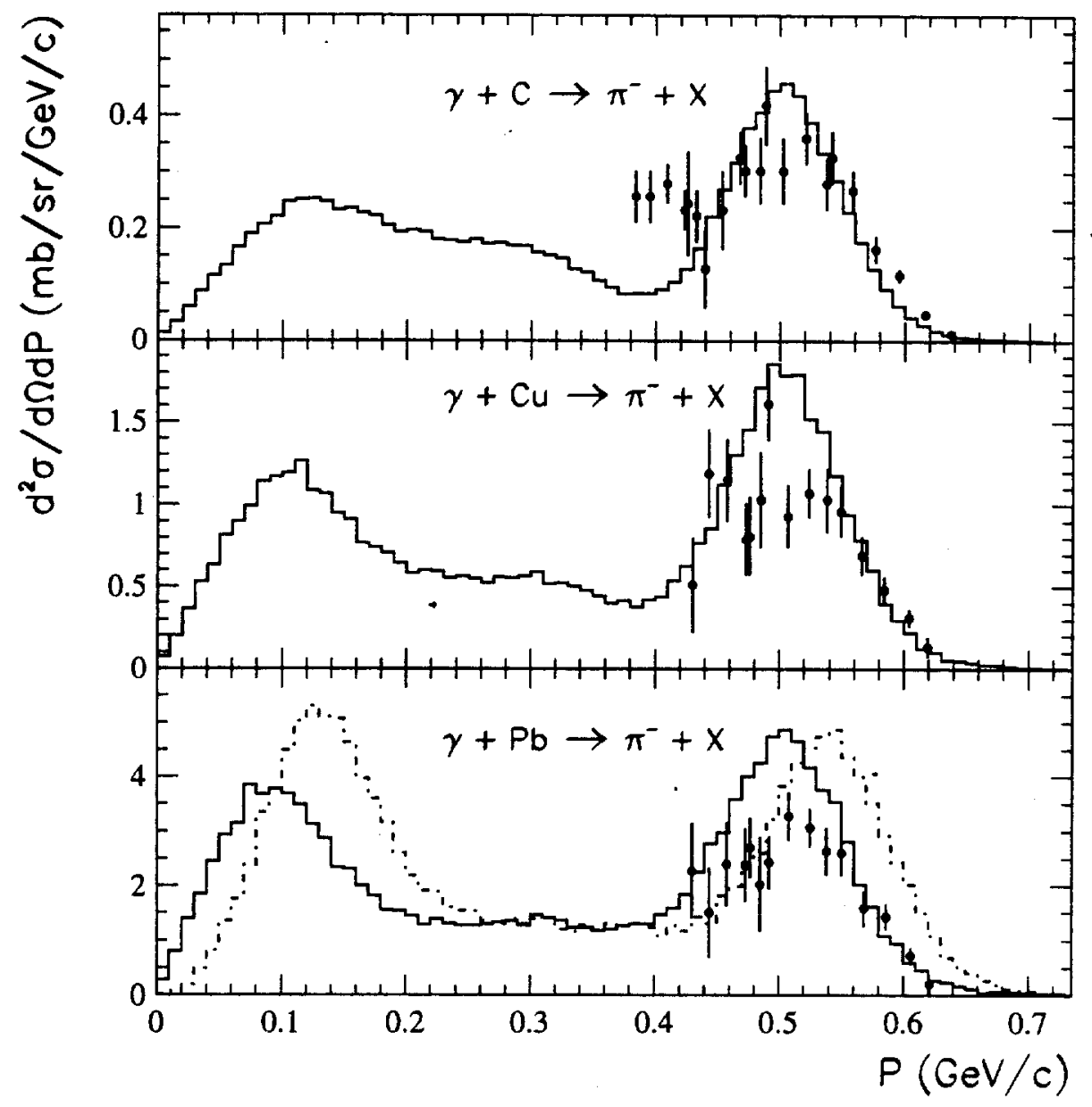

Figure 13: Momentum spectrum of $\pi^{-}$produced on carbon, copper, and lead by $668 \mathrm{MeV}$ photons at the angle $\theta_{\pi}=44^{\circ}$ in the lab.system. The solid histograms are the results of the calculation with the pion potential well depth $V_{\pi}=-35 \mathrm{MeV}$, the dot-dashed histogram, with $\mathrm{V}_{\pi}=0$. The data are from Ref. [35]. 


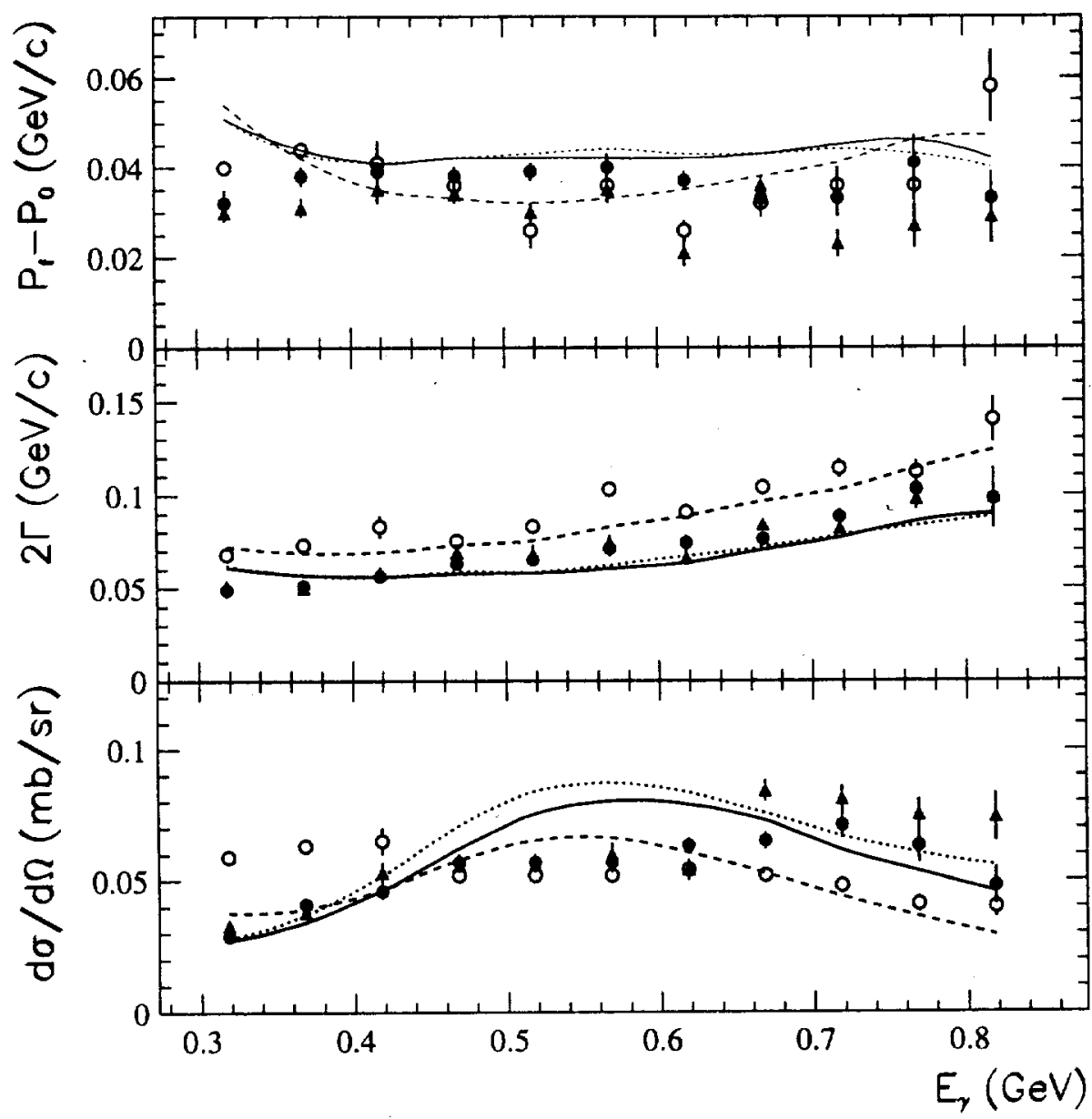

Figure 14: Shift of peak position of quasi-free production of a pion on carbon with respect to its position on a free nucleon (upper part), the peak width (intermediate part), and the peak height (lower part) as a function of the photon energy $E_{\gamma}$. Calculation and experiment are shown respectively by dotted curve and triangles for $\theta_{\pi^{+}}=28^{\circ}$, solid curve and solid circles for $\theta_{\pi^{-}}=28^{\circ}$, dashed curve and open circles for $\theta_{\pi^{-}}=44^{0}$. Points are from Ref. [35]. 


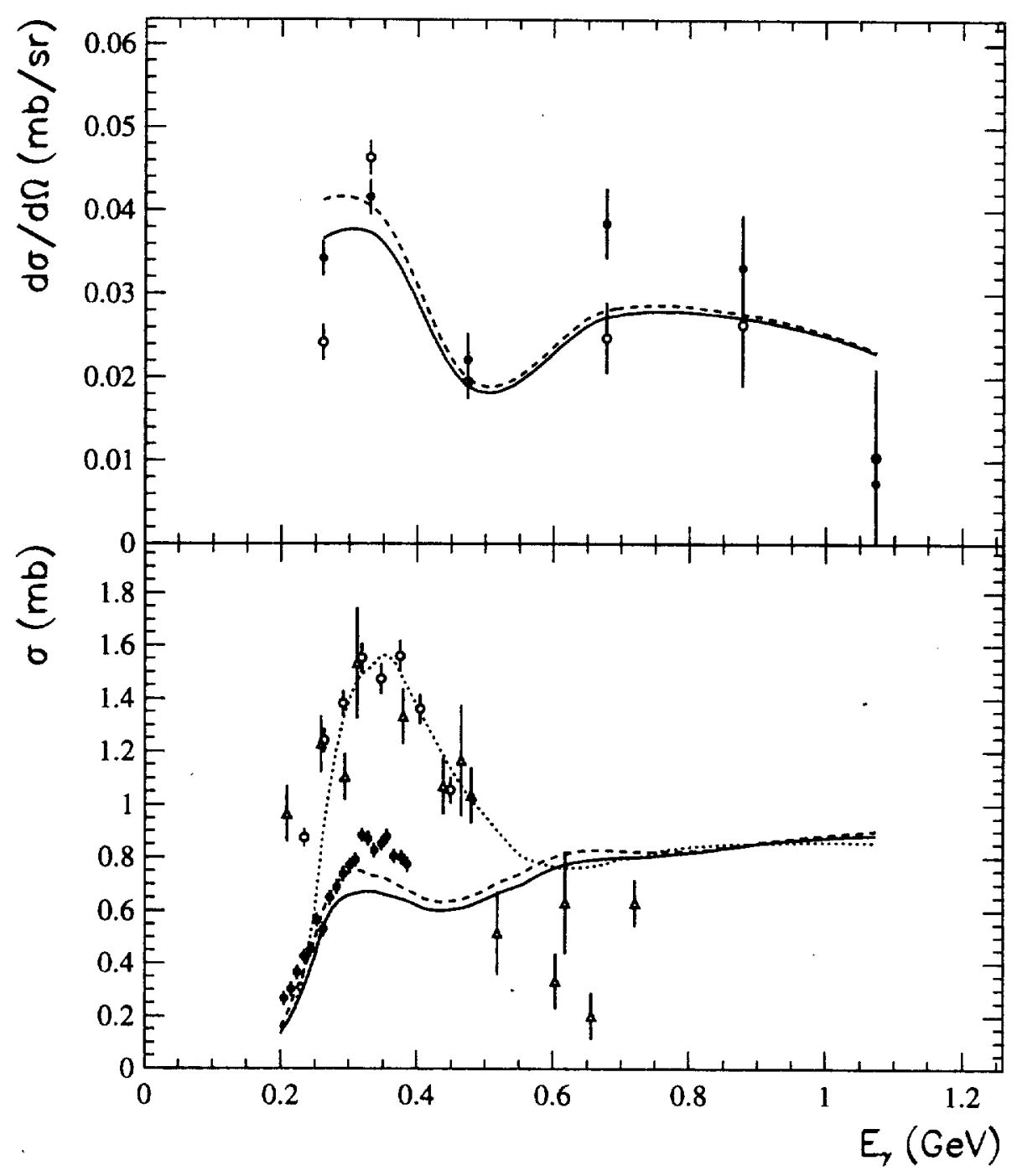

Figure 15: Energy dependence of differential cross section in the lab. system of photoproduction of low energy $\left(0.1 \leq P_{\pi} \leq 0.2 \mathrm{GeV} / \mathrm{c}\right)$ pions at $\theta_{\pi}=90^{\circ}$ (upper plot) and total cross section of pion production on carbon (lower plot). Solid and open circles - data from Ref.[37] for $\pi^{+}$and $\pi^{-}$, respectively (upper plot); solid circles - data from Ref.[3] for $\pi^{+}$; open circles and triangles - data for $\pi^{0}$ from Refs.[38] and [39], respectively (lower plot). Solid, dashed, and dotted curves - calculated results from the Monte Carlo simulation for $\pi^{+}, \pi^{-}$and $\pi^{0}$, respectively. 


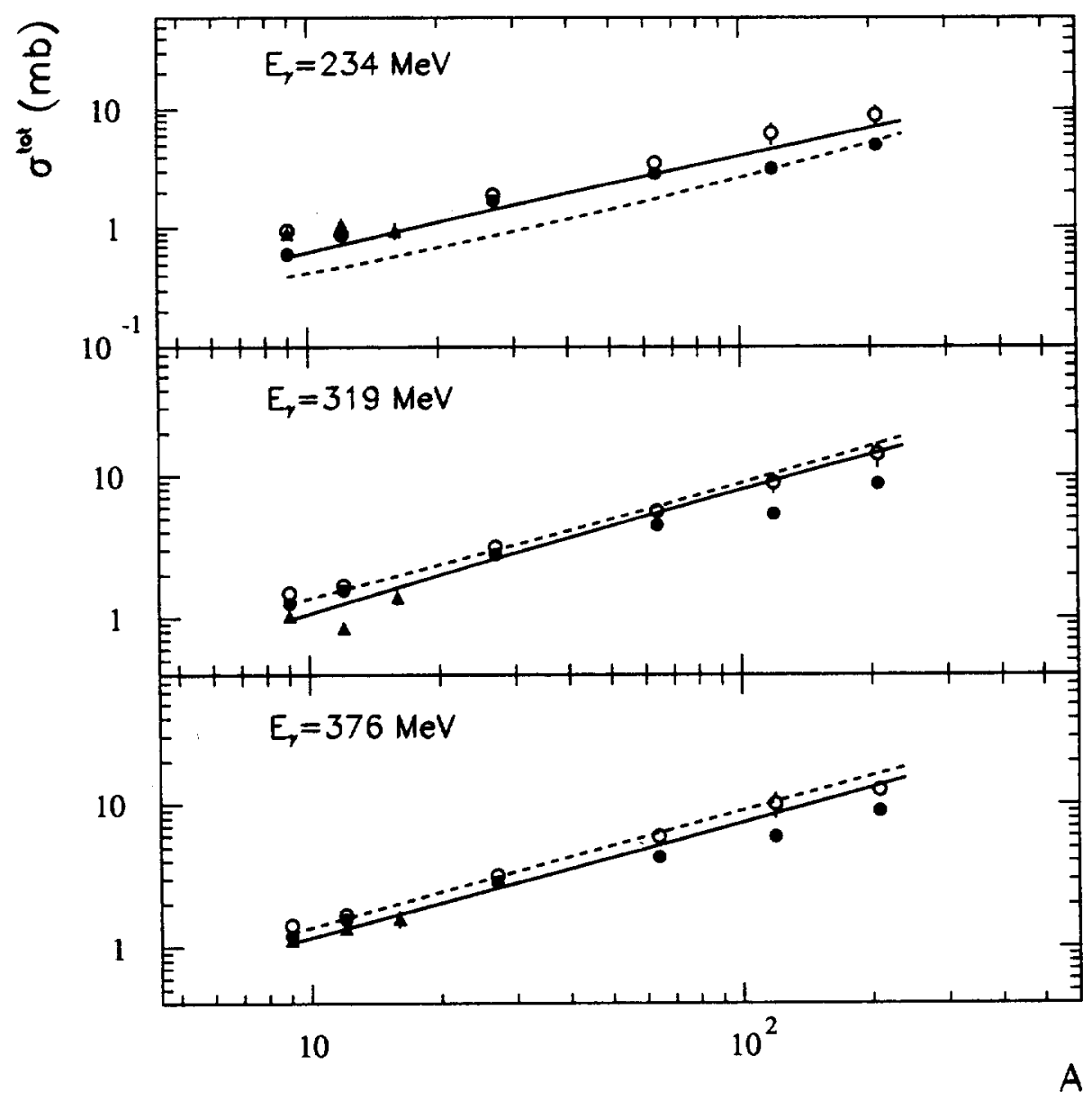

Figure 16: Total cross section of pion photoproduction as a function of nucleon number $A$ of nuclear targets for the given $E_{\gamma}$ values. Open circles - data from Ref.[38] for $\pi^{ \pm}$production; solid circles and triangles - data for $\pi^{0}$ production from Ref. [38] and Ref. [39] (for $E_{\gamma}=208 \pm 44 ; 292 \pm 47 ; 375 \pm 52 \mathrm{MeV}$ ), respectively. Solid and dashed curves - calculated results for $\pi^{ \pm}$and $\pi^{0}$, respectively. 


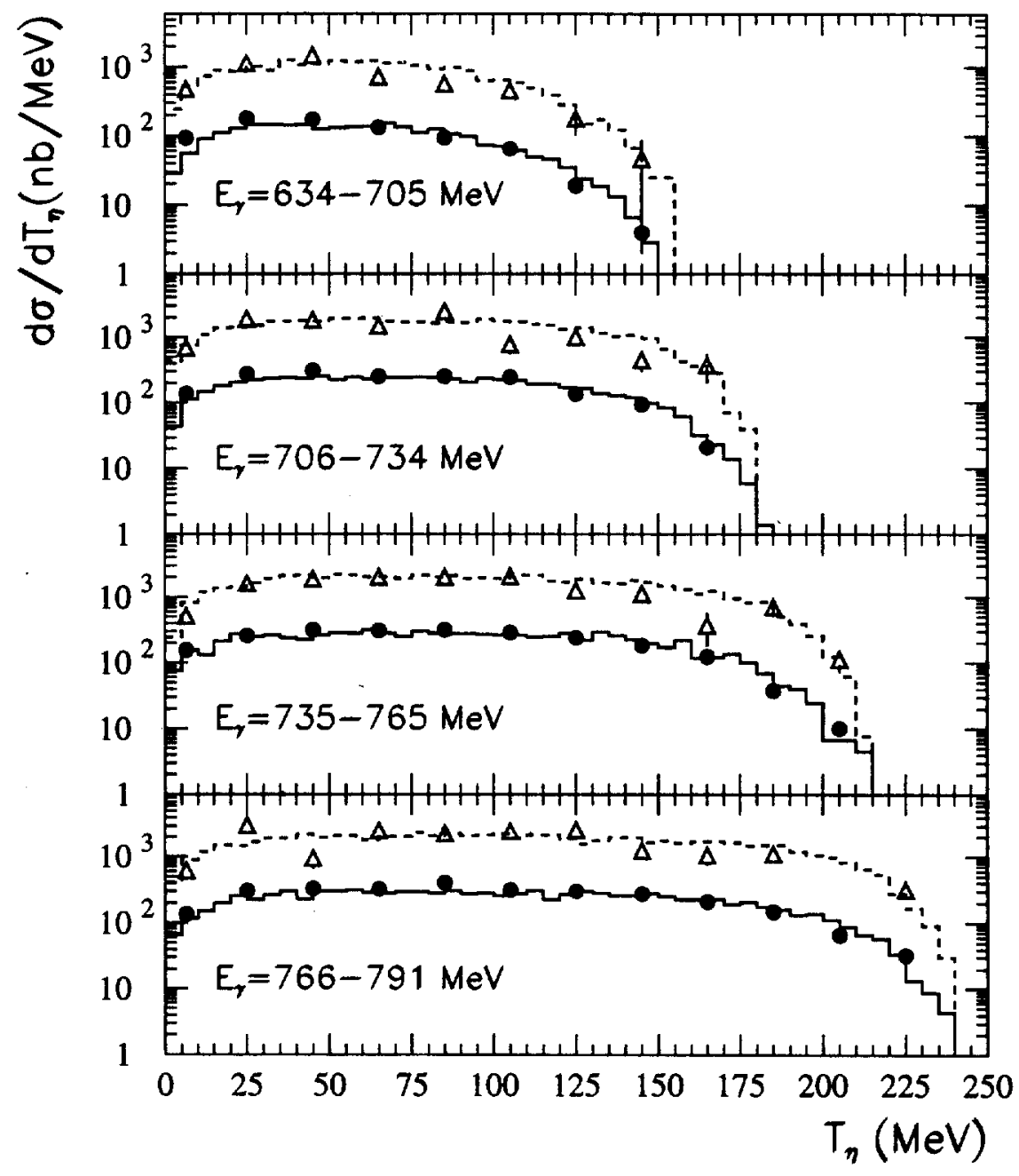

Figure 17: Energy spectra of $\eta$-mesons produced on carbon and lead by photons of the given energy. Calculation and experiment [40] are shown respectively by solid histograms and circles for carbon target, dashed histograms and triangles for lead target. 


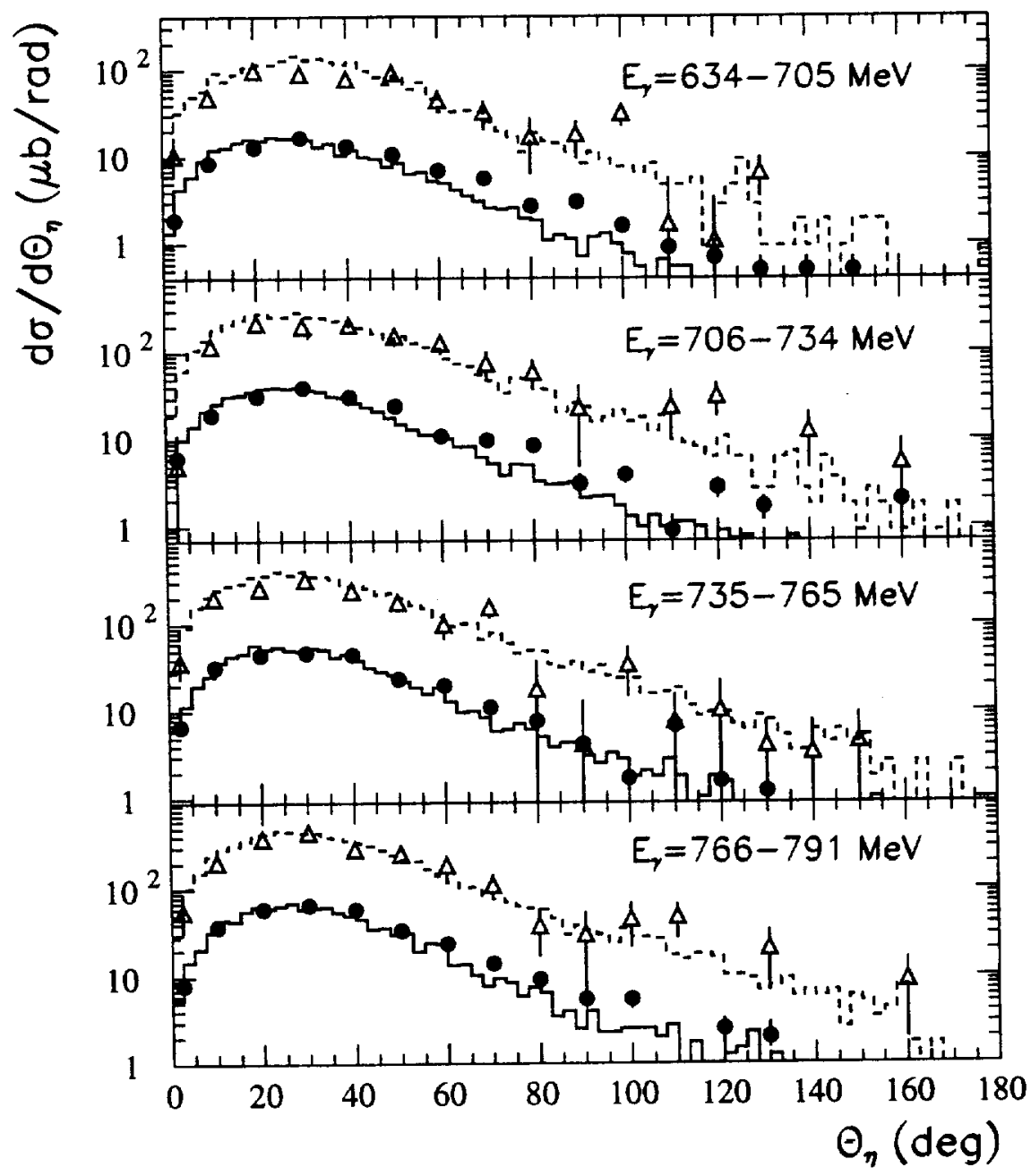

Figure 18: Angular distributions of $\eta$-mesons produced on carbon and lead by photons of the given energy. Notations - as in Fig.17. 
free pion production $\gamma N \rightarrow \pi N$ or $\gamma N \rightarrow i \pi N(i \geq 2)$, its energy is relatively low. But if a proton is emitted from photon absorption on a np-pair, its energy is approximately equal to one half of the photon energy $E_{\gamma}$. Therefore, the high-energy maximum due to the quasideuteron absorption, evident in the spectrum of protons emitted at small angles, transforms to a shoulder and disappears completely with increasing of the angle $\theta_{p}$. The low-energy part of the spectrum is due to protons emitted in the quasi-free pion production, and then it changes weakly with angle. The agreement between theory and experiment is satisfactory taking into account that different measurements deviate from each other by a factor of two in absolute value (see Fig. 19).

The spectrum of protons emitted from heavier nuclei, is formed in a more complicated way. Protons may be emitted in the processes of rescattering of nucleons and pions, two-nucleon absorption of pions, and decay of highly-excited residual nucleus. Fig. 20 shows the $A$-dependence of the differential cross section of proton and pion emission. The INC model predicts a power law dependence, $A^{\beta}$, with $\beta=$ 1.15. This value, larger than the $\alpha$ value for pion production and even larger than 1 , is due to the final state interaction of pions (mainly, pion absorption), which leads to a decreasing of $\alpha$ and to an increasing of $\beta$-value.

Fig. 21 shows the angular distribution of protons emitted in coincidence with neutron in the interaction of photons with carbon. The figure clearly indicates a contribution from the quasi-deuteron mechanism. It should be noted that the role of the two-nucleon photoabsorption mechanism stays practically unexplored in the high energy region $E_{\gamma} \geq 0.5 \mathrm{GeV}$. In particular, the contribution of elementary $\gamma d$ subchannels with pion production is not known. Beside this, in the absorption of high-energy photons, the two nucleons are so close to each other that they may be considered as a 6-quark configuration, and so the probability of photon absorption by $n n$ - and $p p$-pairs may increase considerably.

\section{Conclusions}

We have extended the INC model for photonuclear reactions at energies up to $10 \mathrm{GeV}$. To this end, we have formulated a phenomenological method for exclusive description of the elementary $\gamma N$-interaction taking into account both the resonant contribution from the two-body channels $\gamma N \rightarrow \pi B^{\star}$ and $\gamma N \rightarrow M^{\star} N$ with production of baryon $B^{\star}$ and meson $M^{\star}$ resonances, and the non-resonant statistical contribution from many-body channels $\gamma N \rightarrow i \pi N(2 \leq i \leq 8)$. Moreover, we have taken into account rescattering and absorption in a nucleus of pions, nucleons and $\eta-, \rho-, \omega$-mesons produced in the $\gamma N$-interaction. Finally, we have calculated the evaporation, fission and multifragmentation of highly excited residual nucleus with the initial conditions defined by the INC model. As a result, we have obtained an exclusive description of all products of a $\gamma A$-reaction. So, the INC model can now be used to analyse a wide range of experimental photonuclear data both inclusive and exclusive ones.

The INC model presented in the present paper well describes the available data on the meson and proton production on nuclei by quasi-monochromatic photons. 

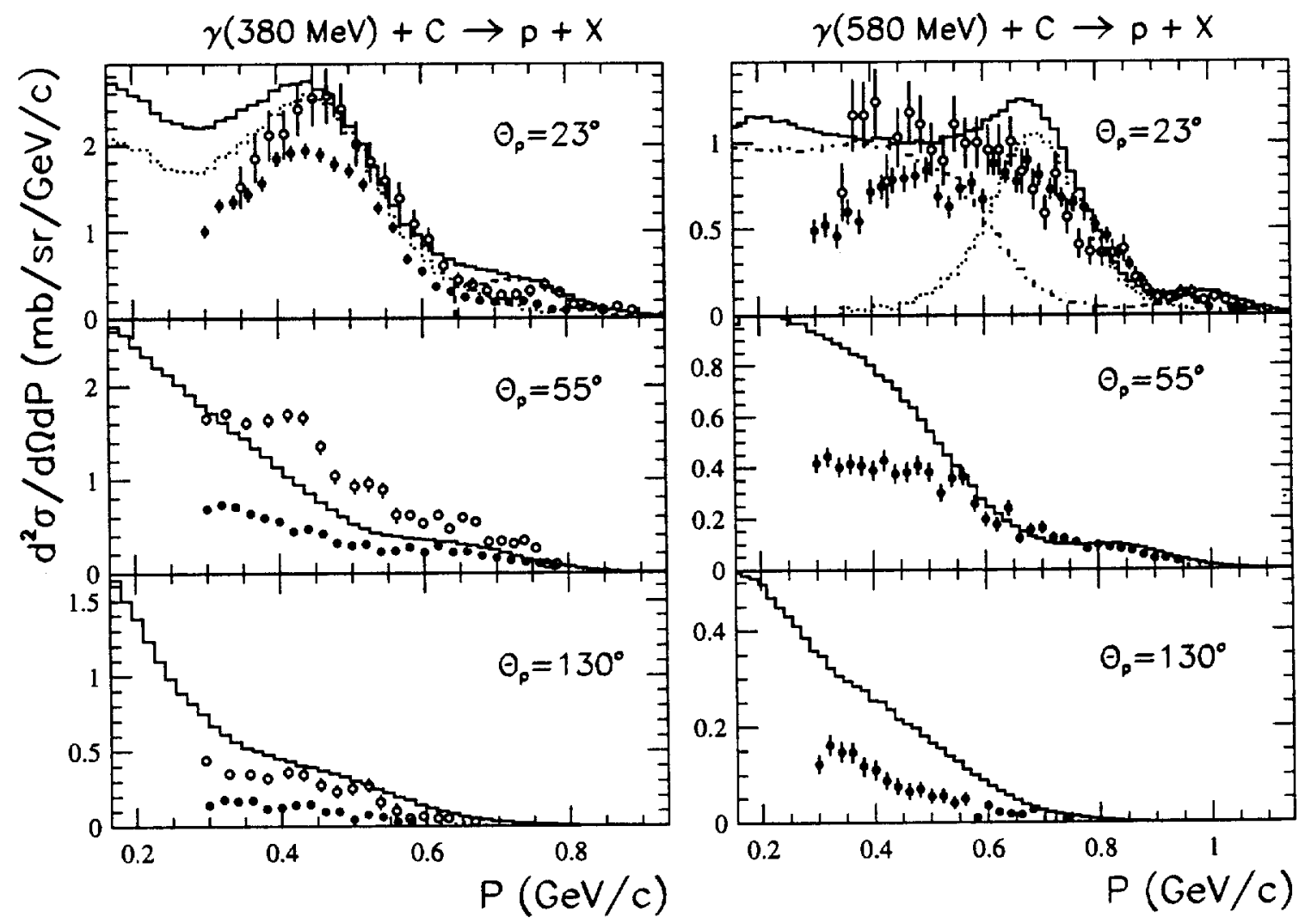

Figure 19: Double differential cross section of photoemission of protons from carbon at $E_{\gamma}=380$ and $580 \mathrm{MeV}$ and angles $\theta_{p}=23^{\circ}, 55^{\circ}$, and $130^{\circ}$. Solid circles - data from Ref.[41], solid histograms - calculated results. Contribution from quasideuteron absorption $\gamma(n p) \rightarrow n p$ is shown by the dashed histogram, contribution from quasifree production of a pion by the dotted histogram, and from multiple pion production by dot-dashed histogram. The data for close photon energies and proton angles ( $E_{\gamma}=397$ and $557 \mathrm{MeV}$ and $25^{\circ} \leq \theta_{p} \leq 30^{\circ}$ from Ref.[42], $E_{\gamma}=380 \mathrm{MeV}$ and $44^{\circ} \leq \theta_{p} \leq 54^{\circ}, 120^{\circ} \leq \theta_{p} \leq 130^{\circ}$ from Ref. [43]) are also shown by open circles. 


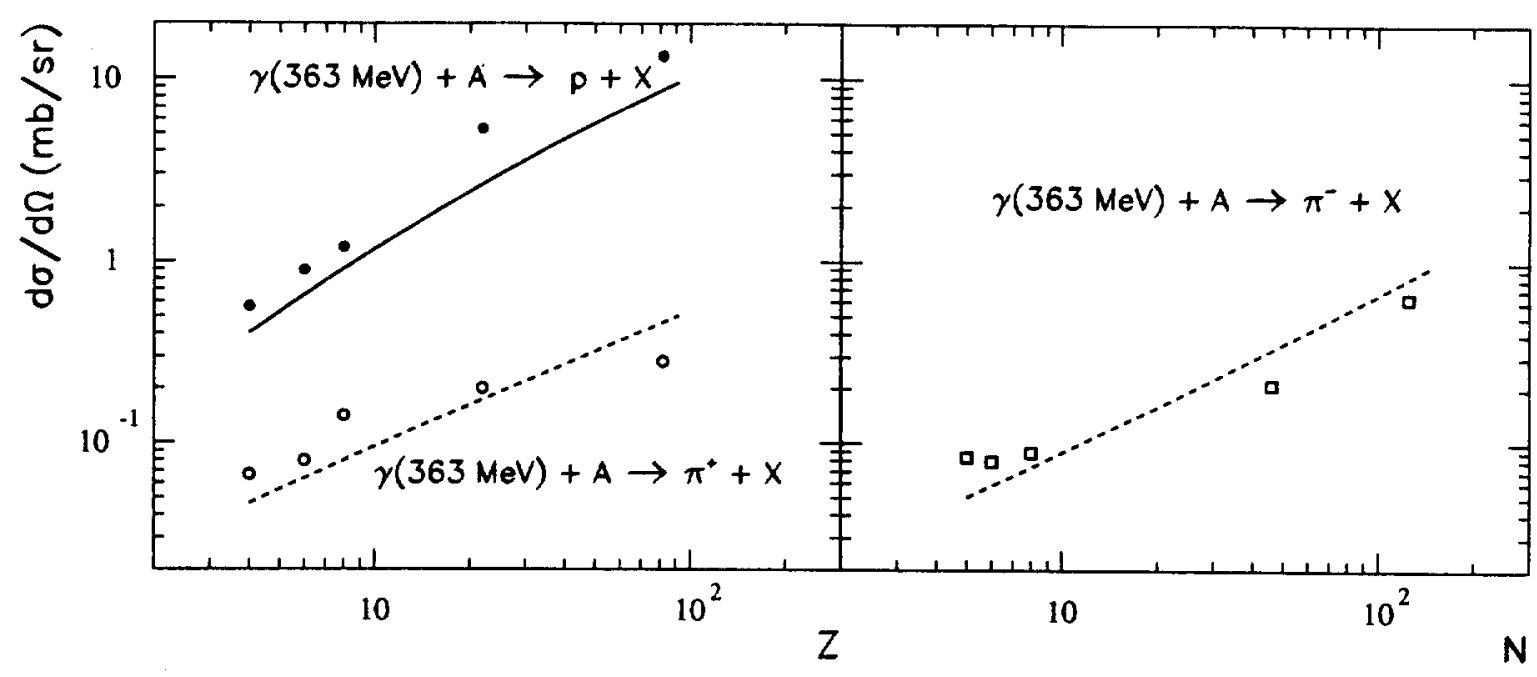

Figure 20: $A$-dependence of the differential cross section of emission of protons and pions at $52^{0}$ in lab. system. Left part: protons and $\pi^{+}$-mesons; right part: $\pi^{-}$mesons. Points - data from Ref. [44]; solid and dashed lines - calculated results for protons and pions, respectively.

Then it can be used to predict and estimate effects, which have not yet been studied in detail, and to simulate photonuclear experiments at new accelerators [45]. Moreover, it can be used for detailed studies on the photoproduction of $\eta_{-}, \rho-$, and $\omega$-mesons on nuclei.

Finally, we would like to mention an analogy with our previous application of the INC model devoted to investigate nuclear reactions initiated by pions, protons or antiprotons $[22],[46]$ and to study the nuclear medium effects in the production of $\omega$ - and $\rho$-mesons in the $\bar{p} \mathrm{~A}$-annihilation [47]. There, it was shown that these effects cause a large increase of width and shift the peak of the mass distribution of the meson resonances. Also the medium effects in photonuclear reactions, that lead to the suppression of baryon resonances in the total photoabsorption cross section on nuclei [48], can be investigated in detail in the framework of the INC model, which is able to simulate the results of coincidence measurements of decay products of $\Delta$ and $N^{\star}$ resonances taking into account the modifications of the properties of baryon resonances in the nuclear medium. Some interesting observations concerning the medium effects would be obtained by comparing these results with corresponding ones of a more sophisticated isobar-hole model [49] which explicitly treats the microscopic medium effects.

Moreover, as it was shown in Ref.[47] for the case of $\bar{p} \mathrm{~A}$ annihilation, the $A$ dependence of the high energy hadron pairs production is rather sensitive to the colour transparency effects. Our INC model can also be used to study these effects in photonuclear reactions, where the high-energy hadron pairs are produced in the $\gamma n \rightarrow \pi^{-} p$ and $\gamma d \rightarrow n p$ elementary processes at high energies $E_{\gamma} \geq 1 \mathrm{GeV}$. 


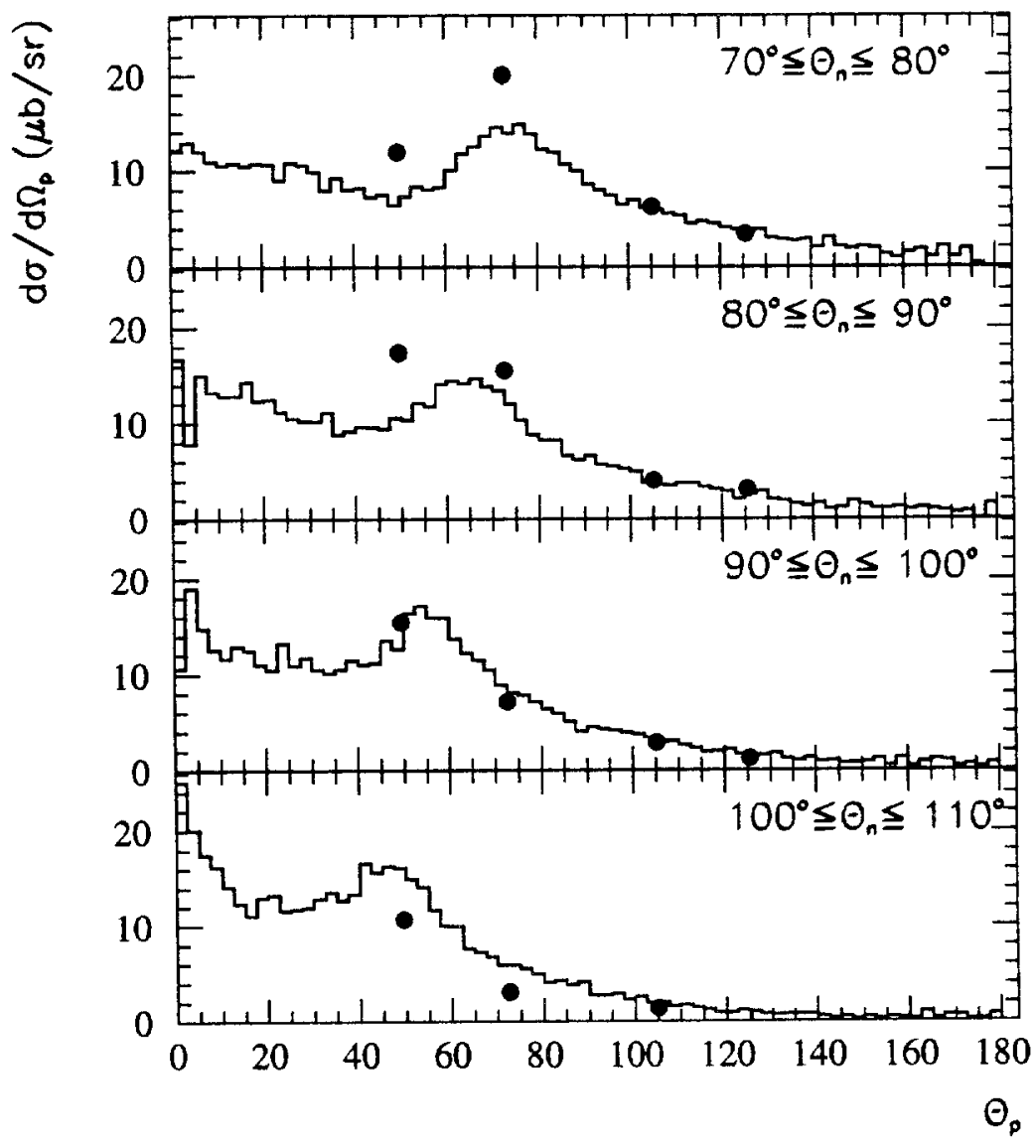

Figure 21: Differential cross section of proton emission in the interaction of photons with energy $315 \leq E_{\gamma} \leq 353 \mathrm{MeV}$ with carbon as a function of the lab. angle $\theta_{p}$ in coincidence with neutron emitted at the given angle $\theta_{n}$ (Energy threshold for protons - $40 \mathrm{MeV}$, for neutrons - $54 \mathrm{MeV}$ ). Points - data from the Ref.[43], histograms - calculated results. 
We thank the INFN-Genova group for providing us with their $\gamma N$-generator code (Ref.[6]). We are grateful to Y.Sumi and B.Krusche for supplying us their experimental data tables. Two of us (A.S. Ijinov and I.A. Pshenichnov) thanks the Laboratori Nazionali di Frascati of INFN for the warm hospitality. I.A.Pshenichnov thanks UNESCO for a short-term fellowship SC/PR201310.3 granted at the initial stage of the present work. The work was supported partially by ISF, grant DEFG05-86ER40285 and by INTAS, grant 93-1560.

\section{References}

[1] K.K. Gudima, A.S. Пjinov, V.D. Toneev, JINR Communications P2-4661, P24808, Dubna, 1969.

V.S. Barashenkov, F. G. Gereghi, A.S. Djinov et al., Nucl. Phys. A231 (1974) 462.

[2] T.A. Gabriel and R.G. Alsmiller, Jr., Phys. Rev. 188 (1969) 1711.

[3] J. Arends, et al., Z. Phys. A305 (1982) 205.

[4] V. Lucherini et al., Phys.Rev. C39 (1989) 911;

A.S. Пjinov et al., Phys.Rev. C39 (1989) 1420;

N. Bianchi et al., Phys. Rev. C48 (1993) 1785.

[5] S.I. Alekhin et al., CERN-HERA 87-01, Geneva, 1987.

[6] P. Corvisiero et al., NIM A346 (1994) 433.

[7] R.L. Walker, Phys. Rev. 182 (1969) 1729;

W.J. Metcalf, R.L. Walker, Nucl. Phys. B76 (1974) 253.

[8] ABBHHM Collaboration, Nucl. Phys. B8 (1968) 535;

D. Menze et al. Compilation of pion photoproduction data. Phys. Inst. der Univ. Bonn, 1977.

[9] T. Fujï et al., Nucl. Phys. B120 (1977) 395.

[10] H. Genzel et al., Z. Phys. 268 (1974) 43.

[11] J.S. Barton et al., Nucl. Phys. B84 (1974) 449.

[12] M. Althoff et al., Z. Phys. C1 (1979) 257.

[13] P. Benz et al., Nucl. Phys. B65 (1973) 158.

[14] M. Ripani, Part. and Field Series, 51 (1993) 418;

M. Ripani et al., CEBAF proposal PR 83-006, 1993.

[15] F. Carbonara et al., Nuovo Cimento A36 (1976) 219.

[16] D. Lüke, P.Söding, Springer Tracts Mod. Phys. 59 (1971) 39. 
[17] B.Krusche et al., Phys.Rev.Lett. 74 (1995) 3736.

[18] D.P.Barber et al., Z. Phys. C2 (1979) 1.

[19] H.R.Hicks et al., Phys. Rev. D7 (1973) 2614.

[20] ABBHHM Collaboration, Phys. Rev. 175 (1968) 1669.

[21] B.Krusche et al., Phys.Lett. B358 (1995) 40.

[22] E.S. Golubeva, A.S. Пjinov, B.V. Krippa, I.A. Pshenichnov. Nucl. Phys. A537 (1992) 393;

I.A.Pshenichnơ, A.S.Ijinov, D.Polster, Phys.Rev.C52 (1995) 947

[23] V.S. Barashenkov, G. M. Zinovjev, Fortshr. Phys. 16 (1969) 48.

Kh. M. Beshtoev, JINR Communications JINR P2-5480, P2-6337, Dubna, 1972.

[24] J. Bystricky et al., Journ. de Phys.(Fr.) 48 (1973) 1901.

[25] A.Braghieri et al., Phys. Lett. B363 (1995) 46.

[26] T.A. Armstrong et al., Phys. Rev. D5 (1972) 1640.

[27] D.O. Caldwell et al., Phys. Rev. D7 (1973) 1362.

[28] M. MacCormik et al., Phys. Rev. C53 (1996) 41.

[29] H. R. Crouch et al., Phys. Rev. 155 (1967) 1477.

[30] P. Rossi et al., Phys. Rev. C40 (1989) 2412.

[31] A.S. Iljinov et al., Intermediate Energy Nuclear Physics. CRC Press, Boca Raton-Ann Arbor-London-Tokyo, 1994.

[32] A.S. Iljinov et al., Nucl. Phys. A543 (1992) 517.

[33] P. Hoffman et al., Phys. Rev. C49 (1993) 2555.

[34] J.P. Bondorf et al., Phys. Reports 257 (1995) 133.

[35] K. Baba et al., Nucl. Phys. A306 (1978) 292; A322 (1979) 349.

[36] A. S. Iljinov et al., Nucl. Phys. A382 (1982) 378; Yad Fiz. 36 (1982) 646.

[37] N. V. Goncharov et al., JETP 64 (1973) 67.

[38] J. Arends et al., Nucl. Phys. A454 (1986) 579.

[39] E.A. Arakelyan et al., Yad Fiz. 42 (1985) 1.

[40] M.Röbig-Landau et al., Phys.Lett. B373 (1996) 45.

[41] T.Takeshita, Journ. Sci. Hiroshima Univ. Ser. A., 45 (1981) 257.

[42] S. Homma et al., Phys. Rev. C27 (1983) 31. 
[43] J. Arends et al., Z. Phys. A298 (1980) 103.

[44] J. Arends et al., Z. Phys. A526 (1991) 479.

[45] N. Bianchi et al., "Photoabsorption and photofission of nuclei". CEBAF experiment 93-019, 1993.

[46] Ye. S. Golubeva et al., Proc. of "2nd Biennial Workshop on $N \bar{N}$ Physics", Moscow, September 13-19, 1993; Phys. of Atomic Nuclei 57 (1994) 1672.

[47] Ye. S. Golubeva et al., Phys.of Atomic Nuclei, 59 (1996) in press No.10.

[48] L. A. Kondratyuk et al., Nucl. Phys. A579 (1994) 453;

N.Bianchi et al., Phys. Rev. C54 (1996) in press No.4.

[49] R. C. Carrasco et al., Nucl. Phys. A536 (1992) 445;

J.A. Gomez Tejedov et al., Nucl. Phys. A588 (1995) 819. 
